\title{
Analysis of Travelling Waves Associated with the Modelling of Aerosolised Skin Grafts
}

\author{
Paula K. Denman ${ }^{\mathrm{a}}$, D. L. Sean McElwain ${ }^{\mathrm{a}}$, John Norbury ${ }^{\mathrm{b}}$ \\ a School of Mathematical Sciences, Queensland University of Technology, GPO Box \\ 2434, Brisbane 4001, Australia \\ ${ }^{\mathrm{b}}$ The Mathematical Institute, Oxford University, 24-29 St Giles, Oxford OX1 3LB, \\ United Kingdom
}

\begin{abstract}
A previous model developed by the authors investigates the growth patterns of keratinocyte cell colonies after they have been applied to a burn site using a spray technique. In this paper we investigate a simplified one-dimensional version of the model. This model yields travelling wave solutions and we analyse the behaviour of the travelling waves. Approximations for the rate of healing and maximum values for both the active healing and the healed cell densities are obtained.
\end{abstract}

Key words: Mathematical modelling, burns, epidermis, skin, wound healing, travelling waves, reaction-diffusion, asymptotic approximation

PACS: 92B05

\section{Introduction}

We have recently developed a mathematical system that models the growth of keratinocyte colonies that have been sprayed onto a burn site (Voges et al., 2006). Here we outline the essential ideas that lead us to the subject of this paper.

When burns are extensive, conventional skin grafts are not adequate to provide wound coverage and other procedures must be used. A current method to assist with skin repair in burn patients is to use cultured epithelial autografts

Email addresses: p.voges@qut.edu.au (Paula K. Denman), s.mcelwain@qut.edu.au (D. L. Sean McElwain), john.norbury@lincoln. oxford.ac.uk (John Norbury). 
(CEA) (Green et al. (1979), O'Connor et al. (1981), Gallico et al. (1984) and DeLuca et al. (1989)). This technique involves taking epidermal cells from an unburnt area of the patient's body and then expanding the skin cells in culture by plating the cells onto a feeder layer of growth-arrested 3T3 cells (a mouse fibroblast cell line). This allows for optimal epidermal cell growth. An epithelial sheet is formed and then grafted onto the patient's burn to provide permanent coverage (O'Connor et al., 1981). This procedure has several disadvantages. It may take 3-4 weeks for the graft to be available (Green, 1991). In addition, the cell sheets are hard to handle as they are very fragile (Harris et al. (1998) and Williamson et al. (1995)). Thirdly, the production of CEA is labor intensive and the process of growing the sheets of cells is expensive (Fraulin et al., 1998). Chalumeau et al. (1999) estimated that the cost of this treatment was approximately US\$80 000 for children and US\$110 000 for adults.

A novel way of delivering epidermal cells is to use a spray apparatus. The paper by Wood (2003) discusses the cultured epithelial autograft suspension that is sprayed onto a wound, CellSpray ${ }^{\circledR}$, that is manufactured by Clinical Cell Culture Ltd in Bentley, Western Australia. Wood has found that cells in suspension are ready for clinical use in as short as five days and, as mentioned above, are a lot easier to handle and administer than CEA in sheet form.

Figure 1 shows the results of experiments carried out at Queensland University of Technology (QUT) where cells have been sprayed onto a prepared surface. The cells have been cultured for 2 hours.

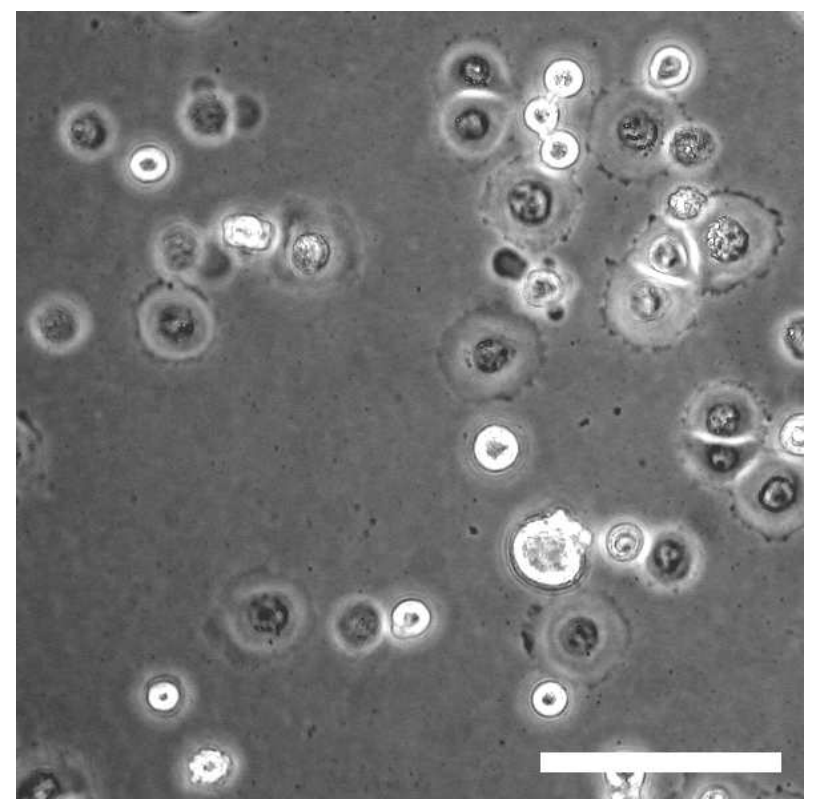

Fig. 1. The distribution of sprayed cells that have been cultured for two hours, from experiments carried out at QUT. This picture is kindly provided by Damien Harkin, un-published data. The bar shown has a distance of $100 \mu \mathrm{m}$.

The model developed previously to simulate the growth of colonies sprayed 
onto the skin consists of a system of partial differential equations (PDE's) that assume the evolution of the cell density can be represented by an extension of the Fisher equation to allow for (a) the keratinocyte cells undergoing a phenotype change to become quiescent and (b) contact inhibition of proliferation. The governing equations are

$$
\begin{aligned}
\frac{\partial u_{1}}{\partial t} & =D_{1} \nabla^{2} u_{1}+k_{1} u_{1}\left(u_{0}-u_{1}-u_{2}-u_{3}-q\right)-\lambda_{1} u_{1} \\
\frac{\partial u_{2}}{\partial t} & =D_{2} \nabla^{2} u_{2}+k_{2} u_{2}\left(u_{0}-u_{1}-u_{2}-u_{3}-q\right)-\lambda_{2} u_{2}+\lambda_{1} u_{1}, \\
\frac{\partial u_{3}}{\partial t} & =D_{3} \nabla^{2} u_{3}+k_{3} u_{3}\left(u_{0}-u_{1}-u_{2}-u_{3}-q\right)-\lambda_{3} u_{3}+\lambda_{2} u_{2}, \\
\frac{\partial q}{\partial t} & =\lambda_{3} u_{3}
\end{aligned}
$$

where $D_{i}$ is the random motility (diffusion) coefficient, $k_{i}$ relates to the rate of proliferation and $\lambda_{i}$ is the rate of conversion $(i=1,2,3)$. The maximum density is $u_{0}$ and space can be occupied by $u_{1}, u_{2}, u_{3}$ or $q$ cells. When cells are sprayed on, it is possible that the cell density is not at its maximum. However, for the purpose of this paper, we assume that the initial clumps are at their maximum density. Here $D_{i}, k_{i}$ and $\lambda_{i}$ are taken to be constants.

There are four different clonal types: highly active, moderately active, least active and quiescent cells. We denote the density of the highly active cells by $u_{1}$ (cells per unit area), the density of the moderately active cells by $u_{2}$, the density of the least active cells by $u_{3}$ and quiescent cell density by $q$.

The equation for the quiescent cell density has a simple form. We are assuming that the quiescent cells do not undergo random motility (migration) and do not proliferate. It can be seen that cell proliferation is assumed to cease when either the space is fully occupied or the cells have become quiescent. There is a transition from a highly active cell to a moderately active cell and then a moderately active cell to a least active cell. The least active cell then becomes quiescent. The reverse transitions are not possible (Barrandon, 1993).

Full details are available in Voges et al. (2006).

While the original modelling is in two dimensions (the burn space) and involves different clonal types, here we investigate a novel model in one dimension and involving only one active species. The governing equations are

$$
\begin{aligned}
& \frac{\partial u}{\partial t}=D \frac{\partial^{2} u}{\partial x^{2}}+k u\left(u_{0}-u-q\right)-\lambda u \\
& \frac{\partial q}{\partial t}=\lambda u
\end{aligned}
$$

where $u(x, t)$ represents the active cell density and $q(x, t)$ represents the quies- 
cent cell density with $x$ representing distance and $t$ representing time. Here $D$ is the random motility of the cells, $k$ is a measure of the proliferative capacity of the cells, $u_{0}$ is the maximum cell density and $\lambda$ characterises the rate at which active cells become quiescent. The initial and boundary conditions are discussed later.

Sherratt (2000) has argued that models of competing close-packed cell populations should include contact inhibition of migration. Here we have followed the approach adopted by Pettet et al. (1996b) and Gaffney et al. (1999) in that we have not included contact inhibition in the random motility terms. Numerical results obtained when Equation (4) below is solved with the term $\frac{\partial^{2} u}{\partial x^{2}}$ replaced by $\frac{\partial}{\partial x}\left((1-u-q) \frac{\partial u}{\partial x}\right)$ are very similar and here we adopt the simpler form for the random motility. This paper is concerned with cell invasion into an empty wound space. If the wound space is occupied by cells of either type then the model could be revised to include contact inhibition explicitly.

In this system, the active cells stop proliferating locally if $u+q=u_{0}$, that is, if the region is fully occupied by active and/or quiescent cells.

In the first part of this paper, we nondimensionalise the equations and present the numerical results for different parameter values showing normal and impaired healing. The numerical solution of the PDE's exhibits travelling wave behaviour. In Section 4 we apply travelling wave analysis to obtain a constraint on the wave speed and numerically solve the travelling wave equations. Section 5 examines four overlapping regions of the solution depending on the dominate terms in the equations to obtain a better understanding of the solution. Section 6 uses an approximation to the travelling wave equations to obtain an approximate solution for $q(x, t)$. This approximate solution is used to obtain a closed form approximate solution. An estimate for the maximum active cell density and the final skin cell density are also obtained. These approximations predict the cell densities to about $1 \%$ accuracy for all values of the model parameter $\alpha>1$. All the tables are located in the Appendix.

\section{Nondimensionalisation}

Before displaying numerical solutions and carrying out the mathematical analysis we nondimensionalise the system. It should be noted that it is possible to write the first equation in the form

$$
\frac{\partial u}{\partial t}=D \frac{\partial^{2} u}{\partial x^{2}}+k u\left(u_{0}-\frac{\lambda}{k}-u-q\right)
$$

although we retain the form (1) for our analysis. 
There are various ways to carry out the nondimensionalisation. Here we scale the cell densities by the maximum density, $u_{0}$, and adopt a timescale that is associated with the rate at which active cells become quiescent. We then obtain

$$
\begin{aligned}
& \frac{\partial u}{\partial t}=\frac{\partial^{2} u}{\partial x^{2}}+\alpha u(1-u-q)-u \\
& \frac{\partial q}{\partial t}=u
\end{aligned}
$$

where $u^{*}=\frac{u}{u_{0}}, q^{*}=\frac{q}{u_{0}}, t^{*}=\lambda t, x^{*}=\frac{x}{L}$ with $L^{2}=\frac{D}{\lambda}$ and $\alpha=\frac{k u_{0}}{\lambda}$. The asterisks have been dropped for notational convenience. The distance scale, $L$, represents the distance that the active cells can migrate, assuming random motility in the characteristic time $t^{*}$. Note that we require $\alpha>1$ for net growth.

We model the ingrowth between two colonies that have been sprayed onto the wound, as shown in Figure 2. We assume that there are no cells in the wound initially so that $u(x, 0)=q(x, 0)=0$ inside the burn where $-l_{2}<x<0$, $l_{2}$ positive, and $u(x, 0)=1$ and $q(x, 0)=0$ for a sprayed cell colony where $0<x<l_{1}$. We assume that the left end of the domain is the burn centre so that we impose a no flux condition there, namely $\frac{\partial u}{\partial x}=0$ at $x=-l_{2}$. We assume that the centre of the colony is at $x=l_{1}$ where we also have a no flux condition so that $\frac{\partial u}{\partial x}=0$ at $x=l_{1}$.

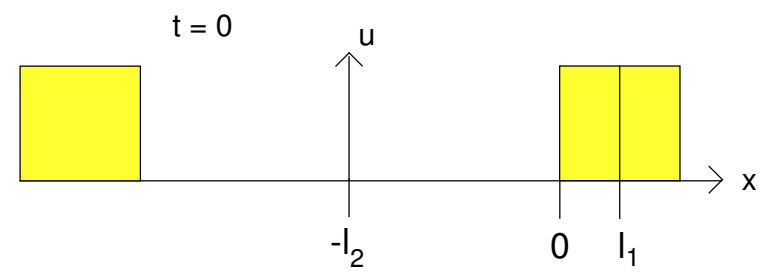

Fig. 2. A schematic diagram of the wound area at $t=0$. Here $-l_{2}$ is the centre of the burn and $l_{1}$ is the centre of the active cell colony.

\section{Numerical Solutions}

Equations (4) and (5) are solved using the method of lines and a finite differences spatial discretisation, as implemented by the NAG Fortran Library routine D03PCF. 
Figure 3 shows the evolution of the active cell density represented by $u(x, t)$ (blue dot-dash line), the quiescent cell density represented by $q(x, t)$ (red dotted line) and the total cell density $(u+q$, solid black line) as a function of $x$ at equally spaced time for the scaled maximum proliferation rates $\alpha=2$ and $\alpha=10$. Active cells migrate into the wound space and proliferate to start to close the wound. These active cells become quiescent and the wound space behind the pulse of active cells is filled with quiescent cells. The region of cells is then occupied by proliferating and/or quiescent cells (solid black line). For certain $\alpha$ values, the cells never completely close the wound, for example $\alpha=2$ as shown in Figure 3(a). Proliferation has stopped and the cells become quiescent, so the wound does not completely fill.

It is interesting to note the similarity of these solutions with those discussed in Pettet et al. (1996a) which models the wound-healing process in soft tissue. In that model, the capillary tips display a chemotactic response to the presence of a chemoattractant. The tip profile is very like the active cell distribution in the model being discussed here.
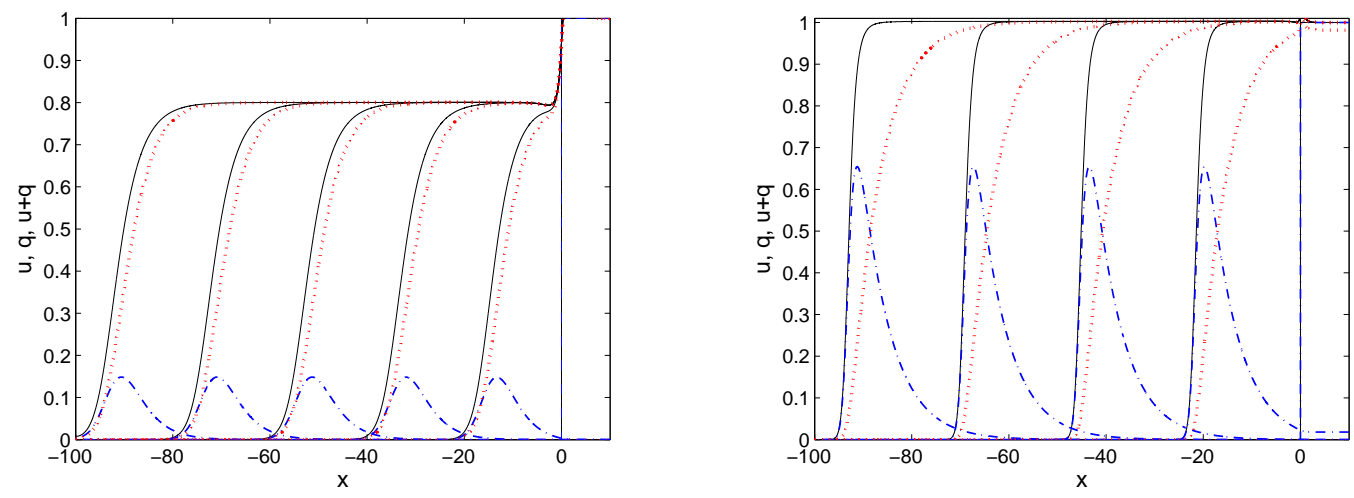

Fig. 3. Typical numerical solutions for $u(x, t), q(x, t)$ and $u(x, t)+q(x, t)$ plotted as a function of $x$ at equally spaced times $t$. The solution for the active species (blue dot-dash line) appears to evolve to a pulse while the solutions for the quiescent cells (red dotted line) and $u+q$ (solid black line) resemble a travelling wave, all moving with constant shape and speed from right to left. (a) Here $\alpha=2$. Every tenth dimensionless time step up to 50 is shown. From the numerical solutions where the wave forms, the maximum of $u$ is about 0.1489 and $q$ tends to approximately 0.8003 . (b) Here $\alpha=10$. Every fourth dimensionless time step up to 16 is shown. From the numerical solutions where the wave forms, the maximum of $u$ is about 0.6547 and $q$ tends to 1.0027, approximately.

In our model we observe that as $\alpha$ increases, the maximum density of the active cells (maximum $u$ ) increases. Another observation is that, as expected, the time to healing is shorter (i.e. healing occurs faster) as $\alpha$ increases. 


\subsection{Impaired Healing}

Figure 4 shows an example with $\alpha=0.9$ which leads to impaired healing. Since $\alpha<1$, the net growth of active cells is negative (the transformation to quiescence exceeds the proliferative growth).
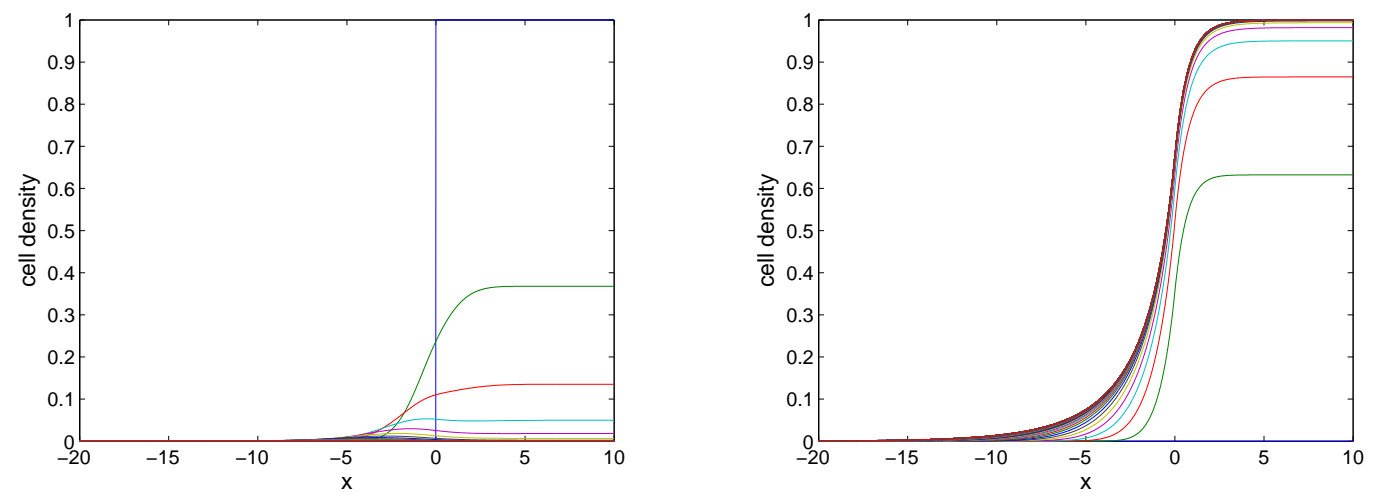

Fig. 4. An example of impaired healing where $\alpha=0.9$ and dimensionless time is to 100. Healing has completely stopped. (a) The distribution of active cells as a function of time. (b) The distribution of quiescent cells as a function of time.

\subsection{Time for the Wound to Heal}

Choosing different values of the maximum scaled proliferation rate, $\alpha$, we can estimate the time it takes for the wound to heal. We assume that the wound is "healed" when the centre of the wound has a cell density of $80 \%$ of the maximum possible cell density. We choose the centre of the wound to be $-l_{2}=-100$. Figure 5 shows a plot of the time to healing against the maximum scaled proliferation rate, $\alpha$ (black solid line). As expected, when the maximum scaled proliferation rate is small, the wound takes a long time to heal and when the maximum scaled proliferation rate is large, the wound heals more quickly.

The time to healing can be determined approximately by the distance the wave has travelled divided by the speed of the travelling wave. The scaled distance of the wound considered is 100 dimensionless units and the dimensionless speed is shown in Section 4 to be approximately $2 \sqrt{\alpha-1}$. Therefore, a good approximation to the healing time is $T_{\left|l_{2}\right|}=\frac{\left|l_{2}\right|}{2 \sqrt{\alpha-1}}+t_{c}$ where $t_{c}$ is the time it takes to set up the travelling wave. The equation $T_{100}=\frac{50}{\sqrt{\alpha-1}}+1$ is shown to be a good approximation (red dotted line) in Figure 5. 


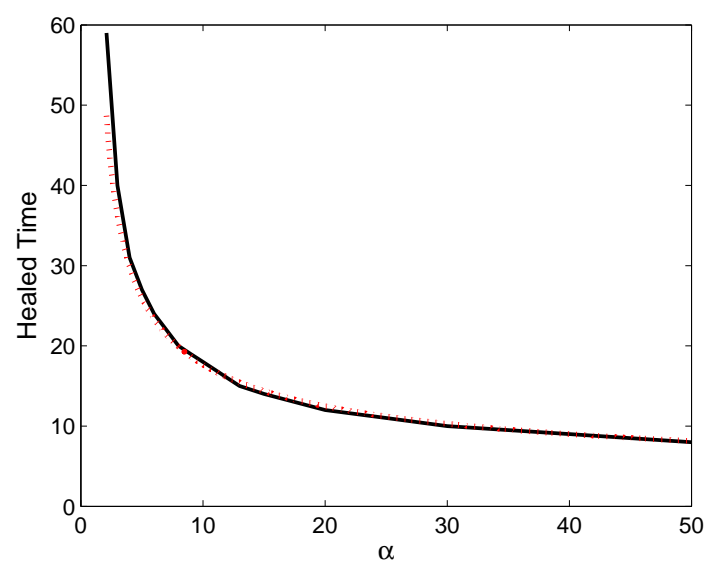

Fig. 5. A plot of the scaled healing time as a function of the scaled maximum proliferation rate, obtained from the numerical solutions (solid black line). The approximation $T_{100}=\frac{50}{\sqrt{\alpha-1}}+1$ is shown as a red dotted line.

\subsection{Limiting Value of the Scaled Quiescent Cell Density}

The limiting value of the scaled quiescent cell density $(q)$, which we denote by $N$, is determined by the time evolution of the quiescent cells. Column one of Table A.2 shows the numerical values of $N$ for different $\alpha$ values. The value of $q$ is the most useful quantity for cell biologists since it corresponds to the healed cell densities.

\section{Travelling Wave Analysis}

The numerical results suggest that the system may, under certain conditions, have travelling wave solutions. We seek a wave of cells moving into the wound with constant shape and speed.

Letting $z=x+a t$, we write $u(x, t)=U(z)$ and $q(x, t)=Q(z)$ where $a$ is the wave speed. Here $a$ is positive since we are considering waves moving to the left. Substituting the solution forms into the system of equations (4) and (5) gives

$$
\begin{aligned}
& a U^{\prime}=U^{\prime \prime}+\alpha U(1-U-Q)-U, \\
& a Q^{\prime}=U,
\end{aligned}
$$

with $U(-\infty)=U(\infty)=Q(-\infty)=0$. Here a prime denotes differentiation with respect to $z$. Recall that $\alpha=k u_{0} / \lambda$ and that we assume $\alpha>1$ to obtain travelling waves. 
We let $S=U^{\prime}$ to obtain the system

$$
\begin{aligned}
S^{\prime} & =a S-\alpha U(1-U-Q)+U, \\
U^{\prime} & =S \\
Q^{\prime} & =\frac{1}{a} U .
\end{aligned}
$$

There are two steady states, the wounded state $(S, U, Q)=(0,0,0)$ and the healed state $(0,0, N)$, where $N$ is arbitrary, and these satisfy the given initial conditions.

We examine the behaviour of the system near the steady states by linearising about the steady states and finding their stability. Set $s_{1}=S, u_{1}=U$ and $q_{1}=Q-\bar{Q}$, where $\bar{Q}=0$ far in front of the wave and $\bar{Q}=N$ far behind the wave.

The linearised equations are

$$
\begin{aligned}
s_{1}^{\prime} & =a s_{1}-\alpha u_{1}(1-\bar{Q})+u_{1}, \\
u_{1}^{\prime} & =s_{1}, \\
q_{1}^{\prime} & =\frac{1}{a} u_{1} .
\end{aligned}
$$

These equations can be integrated by substituting $s_{1}=u_{1}^{\prime}$ and $u_{1}=a q_{1}^{\prime}$ from equations (12) and (13) into equation (11) respectively, for instance, to obtain

$$
s_{1}^{\prime}=a u_{1}^{\prime}-\alpha a q_{1}^{\prime}(1-\bar{Q})+a q_{1}^{\prime} .
$$

This can be integrated with respect to $z$ to obtain the equation

$$
s_{1}=a u_{1}-\alpha a q_{1}(1-\bar{Q})+a q_{1}+c,
$$

where $c$ is a constant of integration. Far in front and far behind the wave, $u_{1}=q_{1}=s_{1}=0$, and therefore $c=0$.

Substituting expression (14) into equation (12) gives the two linear equations

$$
\begin{aligned}
u_{1}^{\prime} & =a u_{1}-\alpha a q_{1}(1-\bar{Q})+a q_{1}, \\
q_{1}^{\prime} & =\frac{1}{a} u_{1} .
\end{aligned}
$$

The eigenvalues of the Jacobian of the linearised system (15) and (16) are given by

$$
\mu=\frac{a}{2} \pm \frac{1}{2} \sqrt{a^{2}-4(\alpha(1-\bar{Q})-1)}
$$


For the wounded steady state $(\bar{U}, \bar{Q})=(0,0)$ the eigenvalues are

$$
\mu=\frac{a}{2} \pm \frac{1}{2} \sqrt{a^{2}-4(\alpha-1)}
$$

We are assuming that $\alpha>1$, therefore, the critical point $(0,0)$ can be classified as:

- an unstable node when $a>2 \sqrt{\alpha-1}$ (two positive unequal eigenvalues),

- an unstable node when $a=2 \sqrt{\alpha-1}$ (one positive repeated eigenvalue), or

- an unstable spiral when $a<2 \sqrt{\alpha-1}$ (a complex conjugate pair of eigenvalues with positive real parts).

We require $u$ and $q$ to be non-negative to be biologically realistic and therefore a spiral solution is not relevant. This gives us a constraint on the speed in front of the wave, namely,

$$
a \geq a_{\min }=2 \sqrt{\alpha-1}
$$

The dimensional form of the wave speed is $a_{\min }=2 \sqrt{D\left(k u_{0}-\lambda\right)}$. For the dimensioned Fisher equation (equations (1) and (2) with $\lambda=0$, so that $q=0$ ), the minimum wave speed is $2 \sqrt{D k u_{0}}$ which is an equivalent result. Thus, the presence of a term that models the transition to quiescence, $\lambda$, slows down the speed of the wave.

To find the approximate scaled wave speed numerically, we follow the technique used in Maini et al. (2004), and the results are shown in Figures 6 and 7. We find the wave speed at the positions $u=0.5, u=0.1$ and $u=0.01$. At each time $t_{i}$, the position of $x_{i}$ where $u$ takes a particular value $(u=0.5, u=0.1$ and $u=0.01$ ) is obtained using linear interpolation between the computed $u$ values. The wave speed is estimated using $\frac{x_{i+1}-x_{i}}{t_{i+1}-t_{i}}$.

As time increases, the wave speed is eventually faster than the minimum wave speed, $a_{\min }=2 \sqrt{\alpha-1}$. Over time the estimates of the wave speed at each of the $u$ positions $(u=0.5, u=0.1$ and $u=0.01)$ converge to the same value, if we have a true travelling wave (unaffected by boundary conditions, etcetera). This behaviour is seen for all $\alpha$ values under the condition that the active cell density, $u$, does in fact reach 0.5 .

For the unwounded steady state $(\bar{U}, \bar{Q})=(0, N)$, the eigenvalues are

$$
\mu=\frac{a}{2} \pm \frac{1}{2} \sqrt{\left.a^{2}-4(\alpha(1-N)-1)\right)} .
$$

The critical point $(0, N)$ can be classified as:

- an unstable node when $a^{2}>4(\alpha(1-N)-1)$ with $N<1-\frac{1}{\alpha}$ (two positive 


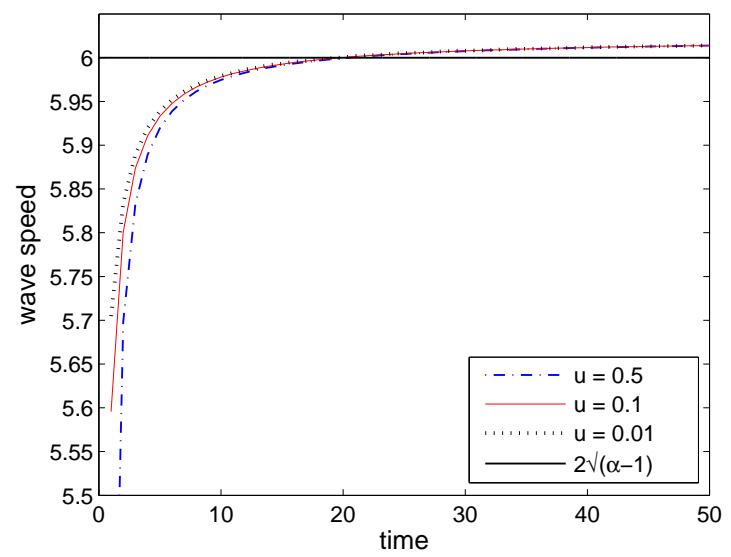

Fig. 6. The numerical wave speeds for when $u=0.5$ (blue dot-dash line), $u=0.1$ (red solid line), $u=0.01$ (black dotted line) and the asymptotic minimum wave speed $a=2 \sqrt{\alpha-1}$ (solid black line) for $\alpha=10$.

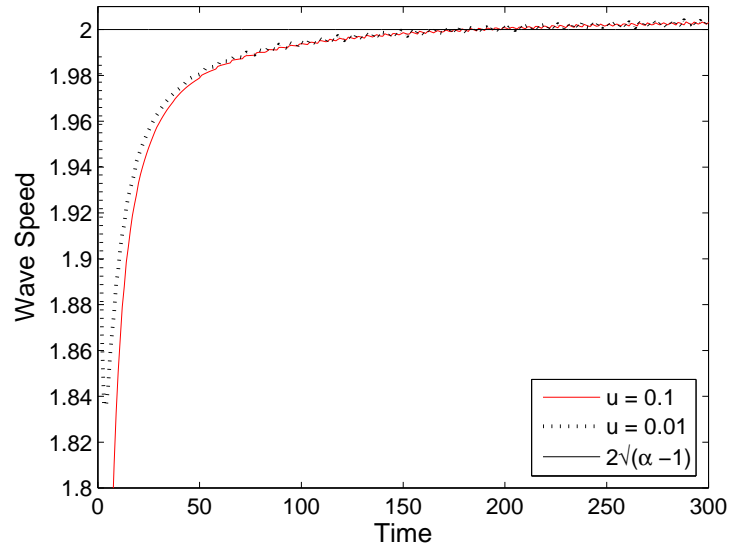

Fig. 7. The numerical wave speeds for when $u=0.1$ (red solid line), $u=0.01$ (black dotted line) and the asymptotic minimum wave speed $a=2 \sqrt{\alpha-1}$ (solid black line) for $\alpha=2$.

unequal eigenvalues),

- a saddle when $a^{2}>4(\alpha(1-N)-1)$ with $N>1-\frac{1}{\alpha}$ (one positive and one negative eigenvalue),

- an unstable node when $a^{2}=4(\alpha(1-N)-1)$ (one positive repeated eigenvalue), or

- an unstable spiral when $a^{2}<4(\alpha(1-N)-1)$ (a complex conjugate pair of eigenvalues with positive real parts).

For the unstable steady state $(0,0)$, the only way a trajectory can be formed to the unstable state $(0, N)$ is in the case of a saddle via the stable separatrix. We therefore require $a^{2}>4(\alpha(1-N)-1)$ and $N>1-\frac{1}{\alpha}$. When $N>1-\frac{1}{\alpha}$ then $a^{2}>4(\alpha(1-N)-1)$ is always satisfied. Therefore, a constraint is that $N>1-\frac{1}{\alpha}$. 


\subsection{Numerical Solutions of the Travelling Wave Equations}

We want to solve

$$
\begin{aligned}
S^{\prime} & =a S-\alpha U(1-U-Q)+U, \\
U^{\prime} & =S, \\
Q^{\prime} & =\frac{1}{a} U,
\end{aligned}
$$

numerically using a shooting method to compare these travelling wave forms with the solutions of the original equations.

In order to determine the behaviour of this system near the steady state $(S, U, Q)=(0,0, N)$ we assume a power series for $Q$ near the steady state, but retain only terms of second degree, in the form $Q(Z)=N-C_{a} Z+C_{b} Z^{2}$ where $Z=e^{\mu z}, C_{a}$ and $C_{b}$ are constants and $\operatorname{Re}(\mu)<0$ because $z \rightarrow \infty$.

Recalling that $U=a Q^{\prime}$, our expression for $U$ is

$$
U=a\left(-\mu C_{a} Z+2 \mu C_{b} Z^{2}\right) .
$$

Substituting our expressions for $U$ and $Q$ into equation (6) and taking the first power in $Z$, we obtain, as expected, $\mu=0$ or $\mu=\frac{a}{2} \pm \frac{1}{2} \sqrt{a^{2}-4(\alpha(1-N)-1)}$.

The second power in $Z$ results in the relationship

$$
4 a \mu^{2} C_{b}=8 C_{b} \mu^{3}-\alpha a \mu^{2} C_{a}^{2}-\alpha \mu C_{a}^{2}+2 \alpha \mu C_{b}\left(1-\frac{1}{\alpha}-N\right),
$$

which can be rearranged to obtain

$$
C_{b}=\frac{-C_{a}^{2} \alpha(a \mu+1)}{2(2 \mu(a-2 \mu)-\alpha(1-N)+1)} .
$$

Having found an expression for $C_{b}$ in terms of $C_{a}$, we can use

$$
\begin{gathered}
Q=N-C_{a} e^{\mu z}-\frac{C_{a}^{2} \alpha(a \mu+1)}{2(2 \mu(a-2 \mu)-\alpha(1-N)+1)} e^{2 \mu z}, \\
U=a\left(-C_{a} \mu e^{\mu z}-\frac{C_{a}^{2} \alpha(a \mu+1)}{(2 \mu(a-2 \mu)-\alpha(1-N)+1)} \mu e^{2 \mu z}\right),
\end{gathered}
$$

and

$$
S=a\left(-C_{a} \mu^{2} e^{\mu z}-2 \frac{C_{a}^{2} \alpha(a \mu+1)}{(2 \mu(a-2 \mu)-\alpha(1-N)+1)} \mu^{2} e^{2 \mu z}\right),
$$

as initial values when shooting from right to left. 
We numerically solve the system of equations (18), (19) and (20) from $z=20$ to $z=-20$ where the initial conditions are given by equations $(21),(22)$ and (23). We choose $\mu=\frac{a}{2}-\frac{1}{2} \sqrt{a^{2}-4(\alpha(1-N)-1)}$ and $C_{a}=0.5$. Since this approximation is only used for $z \rightarrow \infty$, we choose the negative $\mu$ as we require $Z=e^{\mu z}$ to decay as $z \rightarrow \infty$. Here $C_{a}$ is an arbitrarily chosen positive constant. The value of $C_{a}$ varies the position of the wave, but has no effect on the shape of $U$ and $Q$. Our initial starting position, $z=20$ is arbitrarily chosen with the constraints that $z$ is positive (since we chose negative $\mu$ ) and $Z=e^{\mu z}$ is small. Changing the value of $z$, keeping it positive, not too large and not too small, does not change the value of $N$ nor the shape of $U$ and $Q$, just the position of the wave.

Choosing the minimum wave speed (17) and the value of $N$ that allows $U$ and $Q$ to approach zero as $z \rightarrow-\infty$, we solve the system of equations (18), (19) and (20) for different $\alpha$ values. Figure 8 shows the numerical solutions of these equations for $\alpha=2,6$ and 10. In each of the figures, the blue dotted line is the solution for $S$, the green dot-dash line is the solution for $U$ and the solid red line is the solution for $Q$.

Table A.1, shown in the Appendix, compares the maximum of $U$ from the travelling wave solutions with the maximum of $U$ from the PDE numerics for different $\alpha$. For $\alpha \geq 10$ we need to specify $N$ to over 10 decimal place accuracy to shoot successfully. The decimal place accuracy required increases as $\alpha$ increases due to the exponentially small terms as $\alpha$ gets larger. We give the value of $N$ to five decimal places since the numerical solutions are very sensitive to the solution values chosen for large $z$. When integrating, $N$ has to be precise otherwise the values of $U$ and $Q$ do not tend to zero as $z \rightarrow-\infty$.

For $\alpha=4$ and $\alpha=8$, the travelling wave solutions are compared to the solutions to equations (4) and (5) in Figure 9.

Since $Q^{\prime}=\frac{1}{a} U$, we observe that the integral of $U$ from $z=-\infty$ to $z=\infty$ is equal to $a N$, where $N=Q(\infty)$. This shows that, for each $\alpha$, the values of $a$ and $N$ both affect the area under $U$ and hence the shape and height of $U$.

We now want to look at the relationship between the parameter values $a$ and $N$ for a fixed $\alpha$ in equations (18), (19) and (20). For $\alpha=2$ and 10, we vary $a$ and choose $N$ such that $U$ and $Q$ approach zero as $z \rightarrow-\infty$. We use the same initial $z, C_{a}$ and $\mu$ values as above and the initial values given by equations (21), (22) and (23). The length of the domain is determined by the requirement that both $U$ and $Q$ go to approximately zero within the domain. Figure 10(a) shows $N$ versus $a$ for $\alpha=2$ and Figure 10(b) shows $N$ versus $a$ for $\alpha=10$. It can be seen that for a fixed $\alpha$ value there exist solutions for different $a$ and hence $N$. 

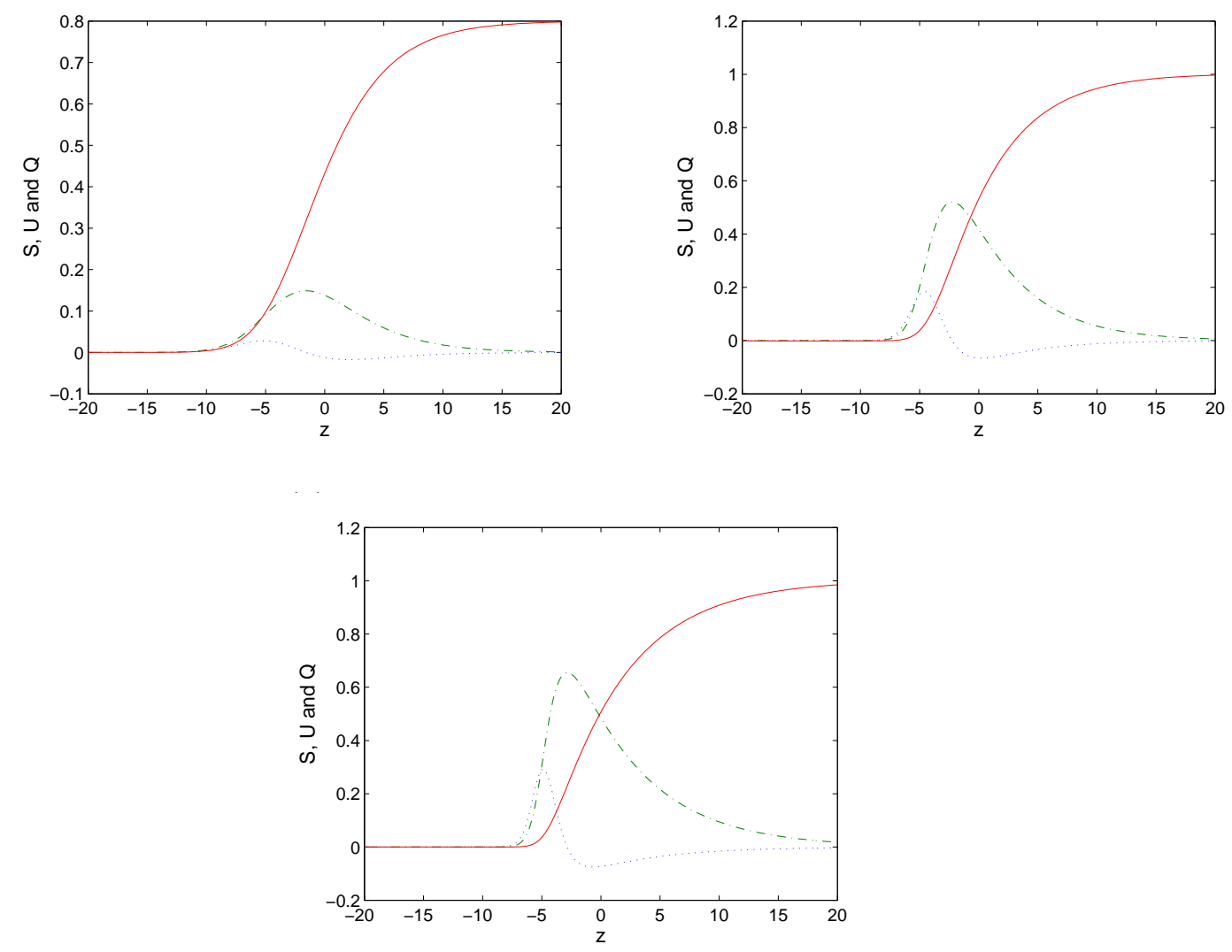

Fig. 8. The numerical solutions of the travelling wave equations. The travelling wave equations are solved on the interval $-20 \leq z \leq 20$. The solution for $S$ is shown in the blue dotted line, $U$ in the green dot-dash line and $Q$ in the red solid line. (a) Here $\alpha=2, a=2$ and $N=0.80023894$. The maximum of $U$ is 0.1487 . (b) Here $\alpha=6, a \approx 4.472$ and $N=1.00335$. The maximum of $U$ is 0.5209 . (c) Here $\alpha=10$, $a=6$ and $N=1.00264556$. The maximum of $U$ is 0.6551 .

Figures 11(a) and (b) show the solutions $S, U$ and $Q$ for $\alpha=2$ and 10, respectively, for different $a$ values. The value of $N$ is then determined such that $S, U$ and $Q$ approach zero as $z \rightarrow-\infty$. Figure 11(a) shows the solutions when $\alpha=2$ for $a=1$ (black), 2 (red) and 4 (blue). Figure 11(b) shows the solutions for $\alpha=10$ and $a=2$ (black), 6 (red) and 10 (blue). For $a<a_{\min }$, $U$ becomes negative before going back to zero. This reinforces the argument above that it is biologically unrealistic to have $a<2 \sqrt{\alpha-1}$. It can be seen from the figures that changing the value of $a$ significantly changes the shape of both $U$ and $Q$. The travelling wave solutions for $U$ and $Q$ have the same shape as the solutions to the PDE's when $a=2$ for $\alpha=2$ in Figure 11(a) and $a=6$ for $\alpha=10$ in Figure 11(b). This figure shows the dependance on the parameters of the solution of the travelling wave ordinary differential equations (ODE's).

In this section we first show how to solve the travelling wave ODE's by using 

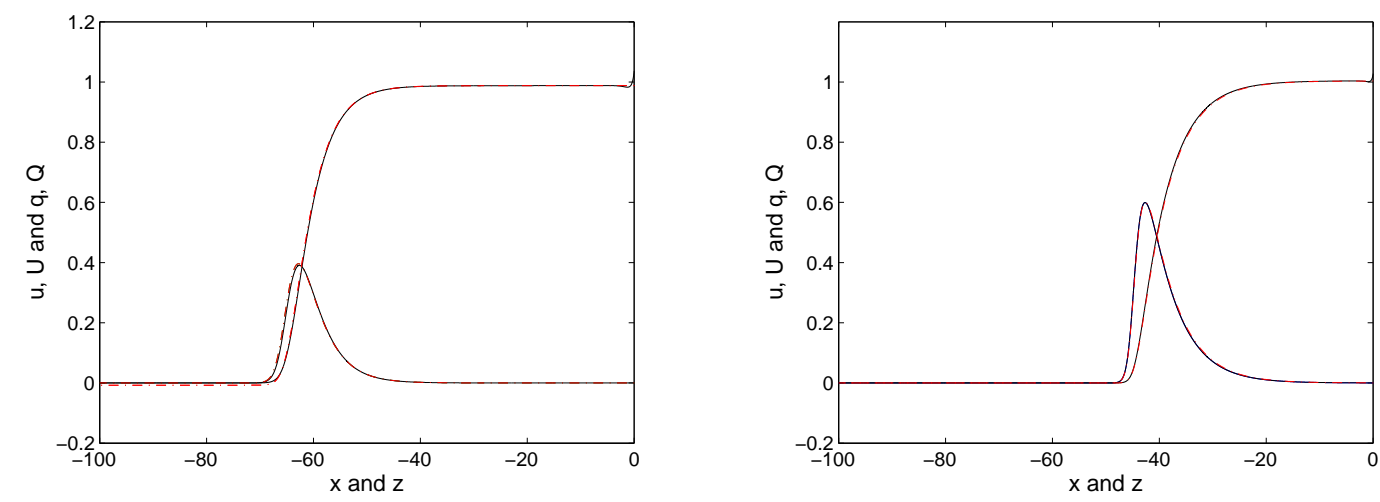

Fig. 9. The numerical solutions of the travelling wave equations (red dot dash line) compared with the solutions to the partial differential equations (4) and (5) (solid black line). (a) Here $\alpha=4, a=2 \sqrt{3}$ and $N=0.9874$. (b) Here $\alpha=8, a=2 \sqrt{7}$ and $N=1.00366$. The PDE numerical solutions are shown for times $t=31$ when $\alpha=4$ and $t=9$ when $\alpha=8$.
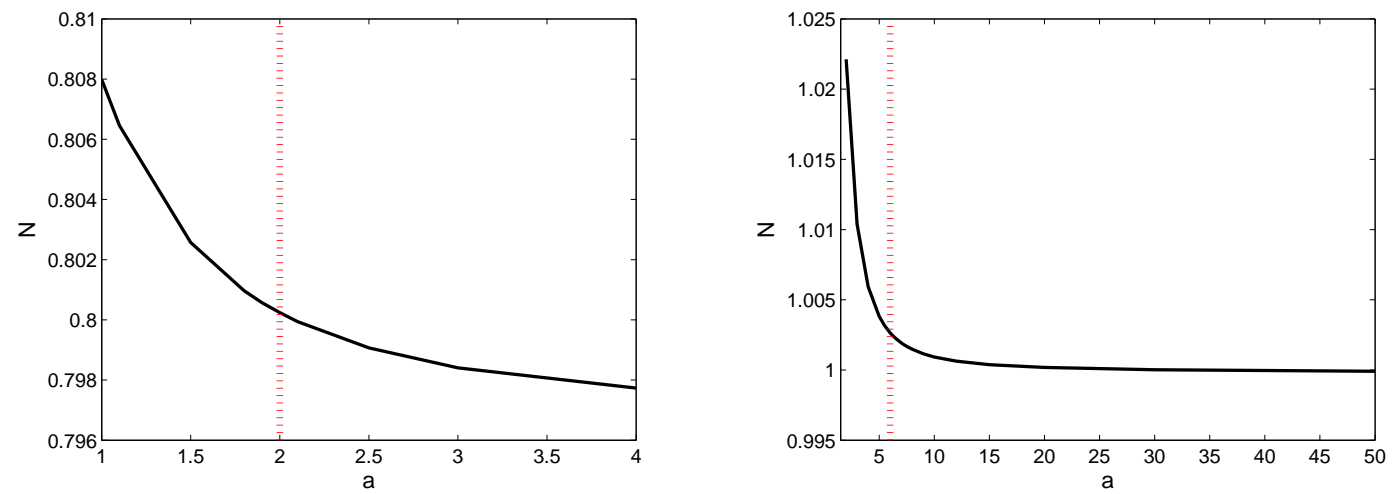

Fig. 10. A graph of $N$ versus $a$. For a given $a$, the corresponding $N$ required for $Q$, $U$ and $S$ from the travelling wave equations to go to zero is shown. (a) The plot for $\alpha=2$. The minimum wave speed of 2 is shown with a red dotted vertical line. (b) The plot for $\alpha=10$. The minimum wave speed of 6 is shown with a red dotted vertical line.

a series expansion to cope with the numerical difficulty of $z \rightarrow \infty$. Then we solve the travelling wave ODE's for a wide choice of parameter values $a$ and $\alpha$. We observe that as the key parameter $a$ is increased, for fixed $\alpha$, we first see a region where the travelling wave ODE solutions are physically unrealistic (negative) and then a region where we get physically realistic solutions. The minimum value of the parameter $a$ where we switch between the above behaviours gives the best fit with the PDE solution that arose from compact initial data. 

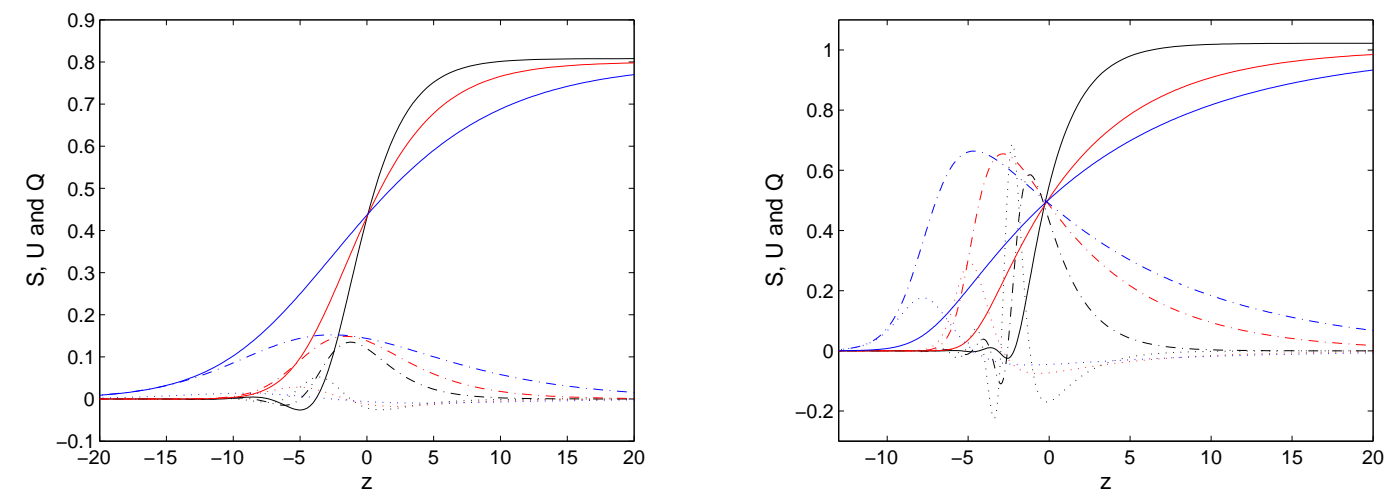

Fig. 11. The travelling wave solutions of $S$ (dotted line), $U$ (dot-dash line) and $Q$ (solid line) for a fixed value of $\alpha$ but with varying $a$ values. Here $N$ is determined such that $S, U$ and $Q$ go to zero as $z \rightarrow-\infty$. The shape and height of $U$ and $Q$ greatly vary depending on the value of $a$. (a) Here $\alpha=2$ and the solutions to $S, U$ and $Q$ are shown for $a=1$ (black), 2 (red) and 4 (blue). For $a=1$, which is below the predicted minimum wave speed of $2, U$ and $Q$ go below zero. The solution when $a=2$ resembles the solution to the PDE's. (b) Here $\alpha=10$ and the solutions to $S$, $U$ and $Q$ are shown for $a=2$ (black), 6 (red) and 10 (blue). For $a=2$, which is below the predicted minimum wave speed of $6, U$ and $Q$ go below zero. The solution when $a=6$ resembles the solution to the PDE's.

\section{Approximate Results from the Travelling Wave Equations for Large $a$}

Performing a shrinking transformation, by rescaling the independent variable $z=a \zeta\left(\zeta=\frac{z}{a}\right)$ in equations (6) and (7), we obtain

$$
\begin{aligned}
\dot{U} & =\frac{1}{a^{2}} \ddot{U}+\alpha U(1-U-Q)-U, \\
\dot{Q} & =U
\end{aligned}
$$

where the notation $\left({ }^{*}\right)$ represents differentiation with respect to $\zeta, U=U(\zeta)$ and $Q=Q(\zeta)$.

Substituting equation (25) into equation (24) we now examine

$$
\frac{1}{a^{2}} \dddot{Q}-\ddot{Q}+\alpha \dot{Q}(1-Q-\dot{Q})-\dot{Q}=0
$$

for $Q(\zeta)$ where $-\infty<\zeta<\infty$ and the parameters $a^{2}, \alpha$ are assumed large. We only consider $a^{2}=4(\alpha-1)$ as this gives the stable PDE travelling wave that we computed. The boundary conditions are $\dot{Q}, \ddot{Q} \rightarrow 0$ as $\zeta \rightarrow \pm \infty$ and $Q \rightarrow 0$ as $\zeta \rightarrow-\infty$. We seek solutions of the form $Q, \dot{Q}>0$ for all $\zeta$ since $Q$ and $U=\dot{Q}$ are cell densities, and define $N:=Q(\infty)=\int_{-\infty}^{\infty} \dot{Q}(\zeta) d \zeta$. 
We expect $Q(\zeta)$ to be as shown in Figures 12 and 13 where we have normalised $\zeta=0$ to be at the maximum of $\dot{Q}$, that is, of $U$. The solution extends through four overlapping regions with distinctive behaviour when $\alpha \gg 1$. These regions are defined by:

(1) $Q, \dot{Q}$ small for $\zeta$ large and negative (linear behaviour),

(2) $Q$ small but $\dot{Q}$ order 1 , and $\frac{1}{a^{2}} \dddot{Q}$ order 1 ; effectively $\zeta<0$,

(3) $Q, \dot{Q}$ order $1, \frac{1}{a^{2}} \dddot{Q}$ small; effectively $\zeta>0$,

(4) $\dot{Q}, \ddot{Q}$ small, $Q$ near $N$ for $\zeta$ large and positive (linear behaviour).

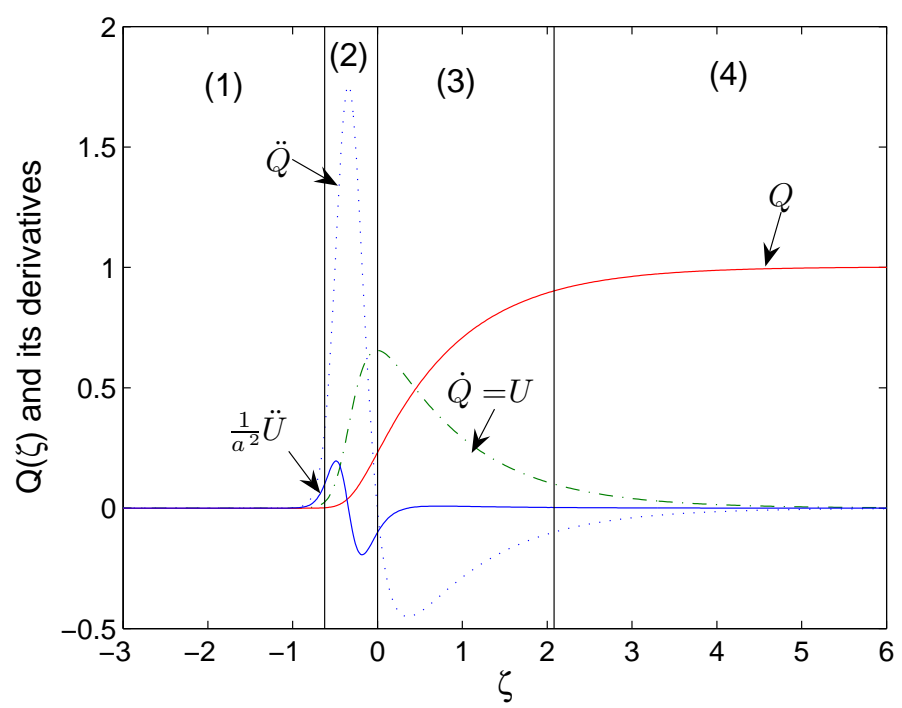

Fig. 12. The solutions for $Q$ (red solid line), $\dot{Q}$ (green dash-dot line), $\ddot{Q}$ (blue dotted line) and $\frac{1}{a^{2}} \dddot{Q}=\frac{1}{a^{2}} \ddot{U}$ (blue solid line) for $\alpha=10$ are shown. The solution $Q$ is divided into four regions, depending on the dominant terms in equation (26).

\subsection{Approximate Solution in Region (1)}

In Region (1) we have $Q$ and $\dot{Q}$ small, resulting in the linearised version of equation (26):

$$
\frac{1}{a^{2}} \dddot{Q}-\ddot{Q}+(\alpha-1) \dot{Q}=0
$$

Solutions of the form $Q=A e^{\hat{\mu} \zeta}$ exist for $\hat{\mu}$ such that

$$
\frac{1}{a^{2}} \hat{\mu}^{3}-\hat{\mu}^{2}+(\alpha-1) \hat{\mu}=0,
$$

where $A$ is a constant and $\operatorname{Re}(\hat{\mu}>0)$ since $\zeta$ is negative. The only positive 


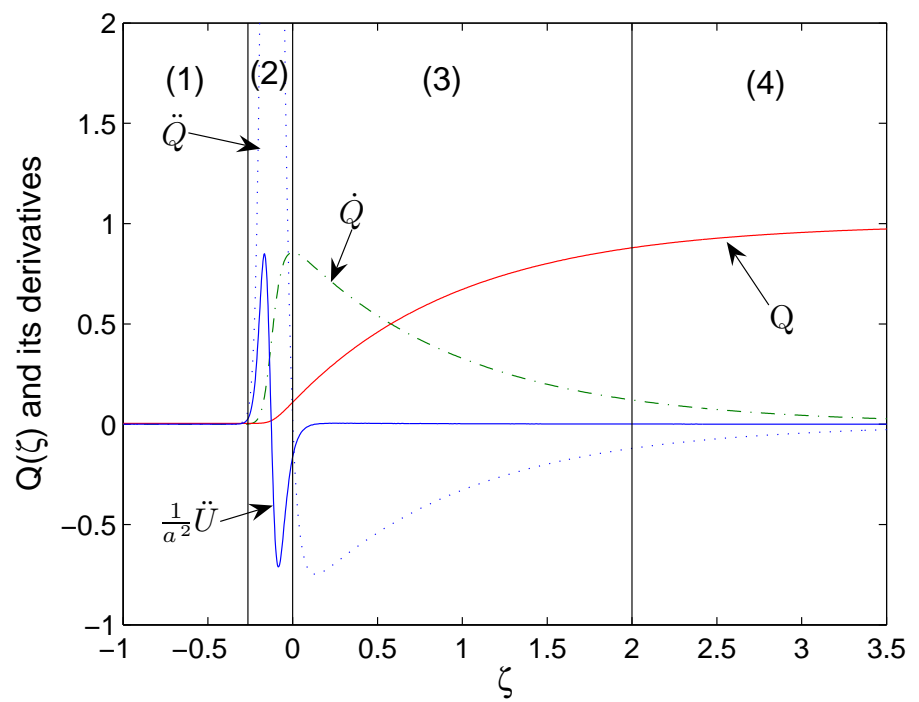

Fig. 13. The solutions for $Q$ (red solid line), $\dot{Q}$ (green dash-dot line), $\ddot{Q}$ (blue dotted line) and $\frac{1}{a^{2}} \dddot{Q}=\frac{1}{a^{2}} \ddot{U}$ (blue solid line) for $\alpha=35$ are shown. The solution $Q$ is divided into four regions, depending on the dominant terms in equation (26). Note that $\ddot{Q}$ goes up to nearly 8 (not shown).

root of equation (28) for $a^{2}=4(\alpha-1)$ is

$$
\hat{\mu}=\frac{1}{2}\left(a^{2} \pm \sqrt{a^{4}-4 a^{2}(\alpha-1)}\right)=\frac{a^{2}}{2} .
$$

For $a^{2}$ large, $A e^{a^{2} \zeta / 2}$ changes significantly over a small length scale $a^{-2}$ in $\zeta$. Thus the non-zero influence in Region (1) will get smaller as $\alpha$ gets larger. In Region (1) the solution of equation (27) is given by

$$
Q=A e^{a^{2} \zeta / 2} \text { with } \dot{Q}=U=\frac{a^{2}}{2} A e^{a^{2} \zeta / 2} \text { and } \ddot{Q}=\frac{a^{4}}{4} A e^{a^{2} \zeta / 2}
$$

Note that $\frac{2}{a^{2}} \dddot{Q}=\ddot{Q}=\frac{a^{2}}{2} \dot{Q}=\frac{a^{4}}{4} A e^{a^{2} \zeta / 2}$ which shows that all the terms in equation (27) are necessary. Also note that $\frac{\dot{Q}}{Q} \rightarrow \frac{a^{2}}{2}$ as $\zeta \rightarrow-\infty$ which will be used later.

\subsection{Approximate Solution in Region (2)}

Since the behaviour shown in (29) forces $\dot{Q}$ to become $O(1)$ before $Q$ does, we can no longer neglect the $\dot{Q}$ term and we hence require Region (2). We drop 
the term involving $Q$ in equation (26) to obtain

$$
\frac{1}{a^{2}} \dddot{Q}-\ddot{Q}+\alpha \dot{Q}(1-\dot{Q})-\dot{Q}=0
$$

Writing $X=\dot{Q}, Y=\ddot{Q}$ we obtain

$$
\begin{aligned}
\dot{X} & =Y, \\
\frac{1}{a^{2}} \dot{Y} & =Y-\alpha X\left(1-\frac{1}{\alpha}-X\right) .
\end{aligned}
$$

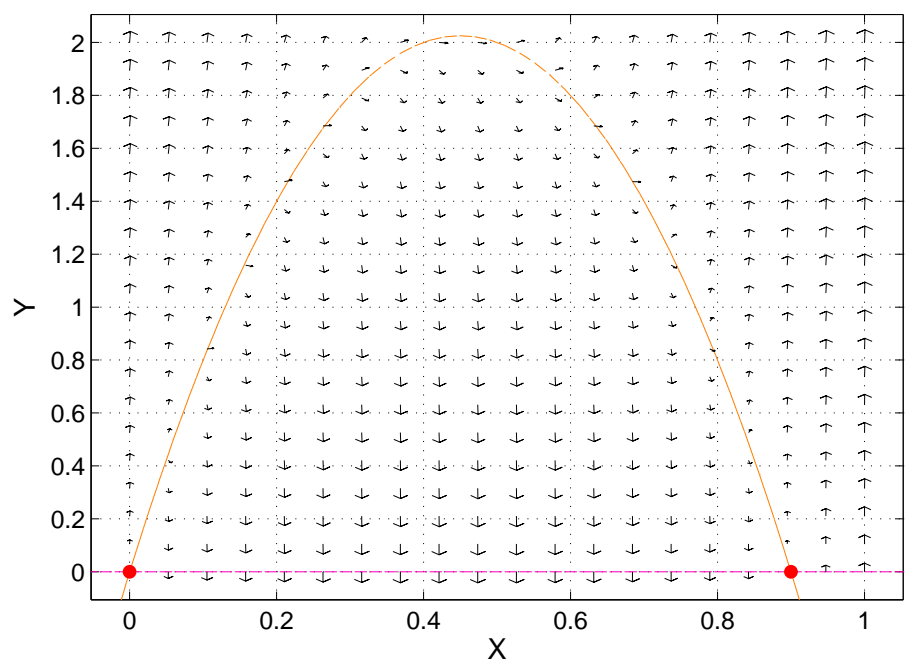

Fig. 14. The $(X, Y)$ phase plane for $\alpha=10$ and $a=6$.

The $(X, Y)$ phase plane system, shown in Figure 14 for $\alpha=10$, has the critical points $(X, Y)=(0,0)$ and $(X, Y)=\left(1-\frac{1}{\alpha}, 0\right)$. We seek an orbit $(X(\zeta), Y(\zeta))$ leaving the node at $(0,0)$ and travelling to the saddle at $\left(1-\frac{1}{\alpha}, 0\right)$. Note that when equations (30) and (31) are solved in linearised form near $X=Y=0$ as $\zeta \rightarrow-\infty$, i.e. as $(X, Y)=(\dot{Q}, \ddot{Q}) \rightarrow(0,0)$, we obtain $\left(\frac{a^{2}}{2}, \frac{a^{4}}{4}\right) A e^{a^{2} \zeta / 2} \rightarrow(0,0)$. By integrating $X$ we see that $\frac{X}{Q} \equiv \frac{\dot{Q}}{Q}=\frac{a^{2}}{2}$ as $\zeta \rightarrow-\infty$, which agrees with Region (1) smoothly.

By looking at the signs of each term in equation (24), we see that at the maximum of $U$, we have $U$ positive, $\dot{U}=0$ and $\ddot{U}$ negative, which forces $U+Q<1$. This also holds in the neighbourhood and to the left of this point. Using $U+Q<1$, in Region (2) we require $Q_{\max }$ to remain less than $1-X_{\max }=1-\left(1-\frac{1}{\alpha}\right)=\frac{1}{\alpha}$ as $U=X \rightarrow X_{\max }=1-\frac{1}{\alpha}$. Note that in Region (2), $Q(\zeta)=\int_{-\infty}^{\zeta} X(\zeta) d \zeta$ increases without bound as we move towards $(X, Y)=\left(1-\frac{1}{\alpha}, 0\right)$ in the $(X, Y)$ phase plane. Since $Q$ should remain less than 
$\frac{1}{\alpha}$ in Region (2), the region must finish before $U \rightarrow 1-\frac{1}{\alpha}$ and we move into Region (3).

Region (2), as shown in Figures 12, 13 and 14, has both $\ddot{Q}$ and $\dddot{Q}$ no longer growing unboundedly as $\zeta$ increases, and then $\dot{Q}$ is drawn towards $1-\frac{1}{\alpha}$ as $\ddot{Q} \rightarrow 0$.

\subsection{Approximate Solution in Region (3)}

In Region (3) we have $Q, \dot{Q}$ bounded and order 1 , and $\frac{1}{a^{2}} \dddot{Q}$ small for $a^{2}$ large, since $\dddot{Q}$ is bounded, so the model equation is

$$
\ddot{Q}=\alpha \dot{Q}(1-Q-\dot{Q})-\dot{Q} \text {. }
$$

We write $\ddot{Q}=\dot{Q} \frac{d \dot{Q}}{d Q}$, so for $\dot{Q} \neq 0$ we have

$$
\frac{d \dot{Q}}{d Q}=\alpha(1-Q-\dot{Q})-1
$$

Then

$$
\dot{Q}=1-Q-C e^{-\alpha Q},
$$

for constant $C$.

For $\zeta$ increasing we want a solution with $\dot{Q} \rightarrow 0$, so that $Q \rightarrow 1$ to this order of approximation (since $\alpha$ large means $e^{-\alpha Q}$ is extremely small, for all constant $C)$. Further, for a constant B,

$$
Q=1+B e^{-\zeta}+\ldots,
$$

as $\zeta \rightarrow \infty$. We need to use Region (4) to find $N=Q(\infty)$ more accurately.

On the other hand, when we consider $\zeta$ decreasing, $Q$ becomes small (note $Q(\zeta)$ is monotonic since $\dot{Q}=U>0$ ). Initially it appears that we could accurately, to leading order, let $Q \rightarrow 0$ and $\dot{Q} \rightarrow 0$ as $\zeta$ decreases to $-\infty$, so that $C=1$. However, this is not asymptotically correct. For $\alpha \gg 1$, if we take $C=1$ in the Region (3) solution, we have $(Q, \dot{Q}) \rightarrow(0,0)$ as $\zeta \rightarrow-\infty$ in our solution, so apparently we do not need Region (2), but if we check $\frac{1}{a^{2}} \dddot{Q}$ we find it is the same size asymptotically (but not numerically) as the biggest terms in equation (32). Further as $Q \rightarrow 0$ we have $\dot{Q}=-Q+\alpha Q+O\left(Q^{2}\right)$, so that $Q=A e^{(\alpha-1) \zeta}=A e^{a^{2} \zeta / 4}$, which contradicts the actual Region (1) behaviour, which is $A e^{a^{2} \zeta / 2}$, as $\zeta \rightarrow-\infty$. Hence, Region (2) is necessary. But the choice $C=1$ turns out to be useful, as we see in Section 6 . 
We now wish to find the maximum of $\dot{Q}$, which occurs at $Q=Q_{*}$, by considering $\frac{d}{d Q}\left(1-Q-C e^{-\alpha Q}\right)=-1+\alpha C e^{\alpha Q_{*}}=0$. Then $\dot{Q}_{\max }=U_{\max }=1-Q_{*}-\frac{1}{\alpha}$ for $Q_{*}=\frac{C \ln \alpha}{\alpha}$. By examining the computed values of the maximum of $u$ from the PDE's (4) and (5), we find that when $C=1.1$, the approximation $U_{\max }$ is accurate to about $1 \%$ for $6 \lesssim \alpha \leq 100$. This is illustrated in Table A.1 in the Appendix.

Hence we obtain

$$
U_{\max } \equiv \dot{Q}_{\max }=1-\frac{1.1 \ln \alpha}{\alpha}-\frac{1}{\alpha},
$$

where we have taken $C=1.1$. This is a good approximation for the maximum of $U$ for large $\alpha$.

Thus, for $\zeta$ decreasing, Region (3) ends when $Q=O\left(\frac{\ln \alpha}{\alpha}\right)$ (and $U=U_{\max }$ ), and we then move into Region (2). To this order we have $Q_{*}=\frac{C \ln \alpha}{\alpha}$ small, so that $\dot{Q}_{\max }=1-\frac{C \ln \alpha}{\alpha}-\frac{1}{\alpha}$ is near $1-\frac{1}{\alpha}$ which gets us "near" the saddle point shown in Figure 14, where $(X, Y)=(\dot{Q}, \ddot{Q})=\left(1-\frac{1}{\alpha}, 0\right)$, for $\alpha$ large. This gives us the correct overlap to match Regions (2) and (3).

\subsection{Approximate Solution in Region (4)}

We finally consider the limit $\zeta \rightarrow \infty$ in equation (26), where not only $\dot{Q} \rightarrow 0$ but $Q \rightarrow N$. In Region (4) our model equation is

$$
\frac{1}{a^{2}} \dddot{Q}-\ddot{Q}+\alpha \dot{Q}(1-N)-\dot{Q}=0
$$

We want a solution $\dot{Q}=-B e^{-\bar{\mu} \zeta}$, where $B$ is a constant, with $\operatorname{Re}(\bar{\mu})>0$, so that

$$
\frac{1}{a^{2}} \bar{\mu}^{2}+\bar{\mu}+\alpha(1-N)-1=0 .
$$

Choosing the positive root of (36) since $\zeta$ is positive, we obtain

$$
\bar{\mu}=\frac{1}{2}\left(-a^{2}+\sqrt{a^{4}-4 a^{2} \alpha(1-N)+4 a^{2}}\right) .
$$

From Region (3) we have $Q=1+B e^{-\zeta}+\ldots$ as $\zeta \rightarrow \infty$, so that to leading order for large $\alpha$ we have $\bar{\mu}=1$ (by matching $B e^{-\zeta}$ ). We define $\lim _{\zeta \rightarrow \infty} Q(\zeta)=$ $N=N_{0}+\bar{N}$ where $N_{0}=1$ from matching with Region (3), and $\bar{N} \rightarrow 0$ as $\alpha \rightarrow \infty$. Then $\alpha(1-N)$ must be asymptotically smaller than 1 so that to leading order $\bar{\mu}=\frac{1}{2}\left(-a^{2}+\sqrt{a^{4}+4 a^{2}}\right)$ (using $a^{2} \gg a^{2} \alpha(1-N)$ ). Next we use the Binomial Theorem to expand the term $\sqrt{a^{4}+4 a^{2}}=a^{2}+2+\ldots$ for $a \gg 1$. Thus $\bar{\mu}=\frac{1}{2}\left(-a^{2}+a^{2}+2+\ldots\right)=1+\ldots$ holds, and we can match onto Region (3) only provided $1-N \ll \frac{1}{\alpha}$, i.e. $N=1+o\left(\frac{1}{\alpha}\right)$. 
We can find the limiting value $N$ more accurately by substituting $Q=1+$ $\bar{N}+B e^{-\zeta}$ into equation (26) to obtain

$$
-\frac{B}{a^{2}} e^{-\zeta}-B e^{-\zeta}-\alpha B e^{-\zeta}\left(B e^{-\zeta}-\bar{N}-B e^{-\zeta}\right)+B e^{-\zeta}=0,
$$

resulting in $\bar{N} \approx \frac{1}{\alpha a^{2}}$. Hence,

$$
N=1+\bar{N} \approx 1+\frac{1}{\alpha a^{2}}
$$

\subsection{The Results from Matching These Regions}

So far we have Region (2) lifting the orbit from Region (1) towards the point $\dot{Q}_{\max }=1-\frac{C \ln \alpha}{\alpha}-\frac{1}{\alpha}$ at $Q_{*}=\frac{C \ln \alpha}{\alpha}$ so that we can match onto Region (3). In Region (3) we can smoothly match onto Region (4) when $N=1+\frac{1}{\alpha a^{2}}$ for $\alpha \gg 1$.

Note that Region (2) is very narrow for large $a$, as we can see from Figures 12 and 13, which implies that the active cells reach their maximum density very quickly, almost before there is any change in the quiescent cell density. Then in Region (3), $\dot{Q}$ decays more slowly while $Q$ increases as the active cells change into healed tissue.

\section{An Approximation to the Travelling Wave Equations}

The rescaling $\zeta=\frac{z}{a}$ of the travelling wave equations, resulting in equations (24) and (25), allows us to neglect the term $\frac{1}{a^{2}} \ddot{U}$ if $a$ is large, to obtain an approximation to the travelling wave equations, namely,

$$
\begin{aligned}
& \dot{U}=\alpha U(1-U-Q)-U, \\
& \dot{Q}=U,
\end{aligned}
$$

where $U=U(\zeta)$ and $Q=Q(\zeta)$. We now examine this model for $\alpha>1$ and $a^{2}=4(\alpha-1)$, even though $a^{2}$ is no longer necessarily large.

This system is the same as previously discussed in 5.3 which has the solution

$$
U=1-Q+C_{2} e^{-\alpha Q},
$$

where $C_{2}$ is a constant.

We now have an approximation for $U$ in terms of $Q$ over the whole domain for $a$ large, which is $U+Q=1$ for $Q \gg \frac{1}{\alpha}$. See Figure 15 for the importance 
of this limit curve $U+Q=1$ which is an envelope for the family of solution curves as $\alpha \rightarrow \infty$.

We know that far in front of the wave, $U=Q=0$ which will give us the condition on $C_{2}$, namely, $C_{2}=-1$. This ensures that the trajectory starts at the origin in this phase plane. Then

$$
U(\zeta)=\frac{d Q}{d \zeta}=1-Q-e^{-\alpha Q}
$$

and

$$
U(z)=a \frac{d Q}{d z}=1-Q-e^{-\alpha Q}
$$

hold in the $(U, Q)$ phase plane with either $\zeta$ or $z$ as the parameter.

Figure 15 shows this phase plane and several trajectories using (39) where $\alpha=50,10,6$ and 2. For $\alpha \leq 1$, the phase portrait has no trajectory for positive $U$.

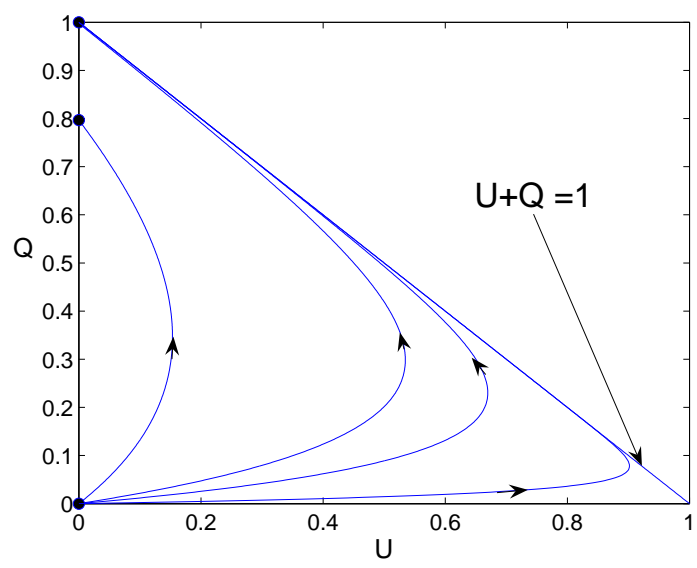

Fig. 15. Phase plane for $(U, Q)$ for the system given by (37) and (38). The parameter $\alpha$ takes the values $50,10,6$, and 2 and the steady states are marked $(\bullet)$. The plot corresponding to the smallest $\alpha$ value has the smallest maximum value of $U$.

The numerical solutions of the approximation (40) are a good fit for all $\alpha$ values. Figure 16 shows the numerical solutions to equations (4) and (5) (black solid line) compared with the numerical solution to the approximation (red dot-dashed line) for $\alpha=1.1,2$ and 50 .

\subsection{Approximate Analytical Solution of $\dot{Q}=1-Q-e^{-\alpha Q}$}

To find a closed form approximate solution for $Q$ and hence $U$ we analyse the approximate equation $\dot{Q}=1-Q-e^{-\alpha Q}$. This cannot be solved explicitly. For $\alpha Q$ large, $e^{-\alpha Q}$ tends to zero, and the equation $\dot{Q}=1-Q$ can be solved 

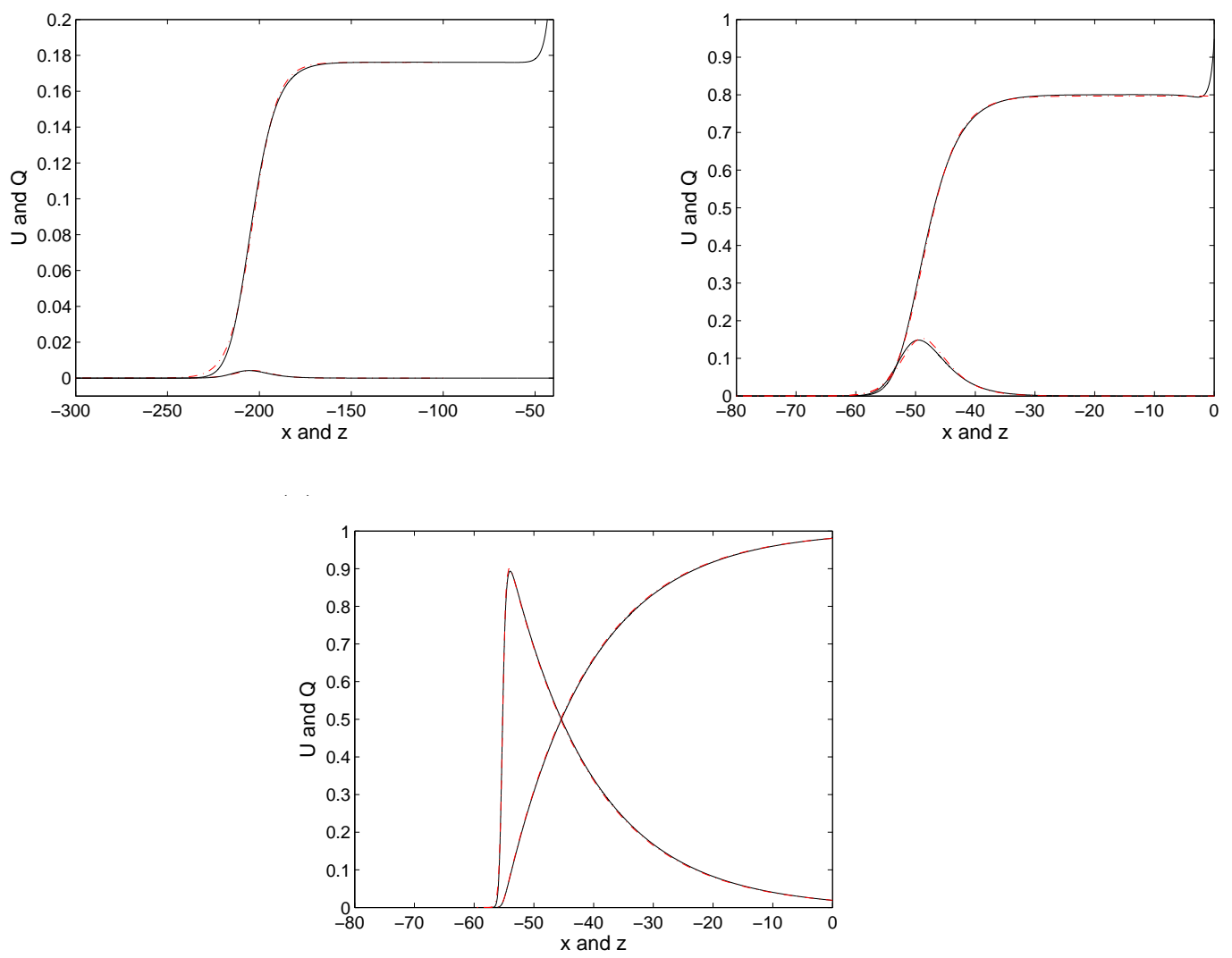

Fig. 16. Numerical results of the PDE's (4) and (5) for $u$ and $q$ are shown in solid black lines. These are compared to the numerical solutions to (40) which are shown in red dot-dash lines. (a) Here $\alpha=1.1$ and $a \approx 0.6325$ and the time for the system of equations (4) and (5) is $t=300$. (b) Here $\alpha=2$ and $a=2$ and the time for the system of equations (4) and (5) is $t=30$. (c) Here $\alpha=50$ and $a=14$ and the time for the system of equations (4) and (5) is $t=5$.

in closed form. For $\alpha Q$ close to zero, a Maclaurin expansion for $e^{-\alpha Q}$ for $\alpha Q$ small can be used to get an approximation.

The solution to $\dot{Q}=1-Q$ is $Q=1-C_{3} e^{-\zeta}$ where $C_{3}$ is a constant. Setting an arbitrary value for $Q(\zeta=0), Q_{0}$, our solution then becomes $Q=1-(1-$ $\left.Q_{0}\right) e^{-\zeta}$

We obtain an approximate equation using a Maclaurin expansion for $\alpha Q$ small, only retaining quadratic terms, namely

$$
\dot{Q}=(\alpha-1) Q-\frac{1}{2} Q^{2} \alpha^{2}
$$


Solving this first order differential equation results in

$$
Q=\frac{2(\alpha-1)}{\alpha^{2}+2 C_{4}(\alpha-1) e^{-(\alpha-1) \zeta}}
$$

where $C_{4}$ is the constant of integration.

Again, taking $Q(0)=Q_{0}$, we obtain $C_{4}=\frac{\frac{2}{Q_{0}}(\alpha-1)-\alpha^{2}}{2(\alpha-1)}$. We require $C_{4}$ to be positive since if $C_{4}<0$, the solution is unbounded for finite $\zeta$. For $C_{4}$ to be positive, we require $Q_{0}<\frac{2(\alpha-1)}{\alpha^{2}}$.

Substituting the expression for $C_{4}$ into the expression for $Q$, we obtain

$$
Q=\frac{2(\alpha-1)}{\alpha^{2}+\left(\frac{2}{Q_{0}}(\alpha-1)-\alpha^{2}\right) e^{-(\alpha-1) \zeta}}
$$

As we require $Q_{0}<\frac{2(\alpha-1)}{\alpha^{2}}$, we choose $Q_{0}=\frac{1}{\alpha}$ for $\alpha>2$. The approximation for $Q$ we obtain is

$$
Q(\zeta)= \begin{cases}\frac{2(\alpha-1)}{\alpha^{2}+(\alpha(\alpha-2)) e^{-(\alpha-1) \zeta},}, & \text { if } Q \leq \frac{1}{\alpha} \\ 1-\left(1-\frac{1}{\alpha}\right) e^{-\zeta}, & \text { if } Q \geq \frac{1}{\alpha}\end{cases}
$$

and thus

$$
U(\zeta)= \begin{cases}1-\frac{2(\alpha-1)}{\alpha^{2}+\left(\alpha(\alpha-2) e^{-(\alpha-1) \zeta}\right.}-e^{-\alpha\left(\frac{2(\alpha-1)}{\alpha^{2}+(\alpha(\alpha-2)) e^{-(\alpha-1) \zeta}}\right)}, & \text { if } Q \leq \frac{1}{\alpha} \\ \left(1-\frac{1}{\alpha}\right) e^{-\zeta}-e^{-\alpha\left(1-\left(1-\frac{1}{\alpha}\right) e^{-\zeta}\right)}, & \text { if } Q \geq \frac{1}{\alpha}\end{cases}
$$

Figure 17 shows the numerical solution of $Q$ from (40) for $Q(0)=10^{-8}$ (shown in dotted red) compared with the analytical approximation of $Q$, expression (43) (shown in solid black). Recall that $\zeta=\frac{z}{a}$. This figure also shows the approximation for $U$ given by equation (40) (shown in dotted red) and (44), the analytical solution to $U$ (shown in solid black). Here $\alpha=2,10$ and 50 and $a=2 \sqrt{\alpha-1}$. For $\alpha=2$ the approximation for large $\alpha Q$ where $z>0$ is not accurate since $\alpha$ is not large. This can be seen in Figure 17(a). We choose $Q_{0}=\frac{1}{2 \alpha}$ for $\alpha=2$. 

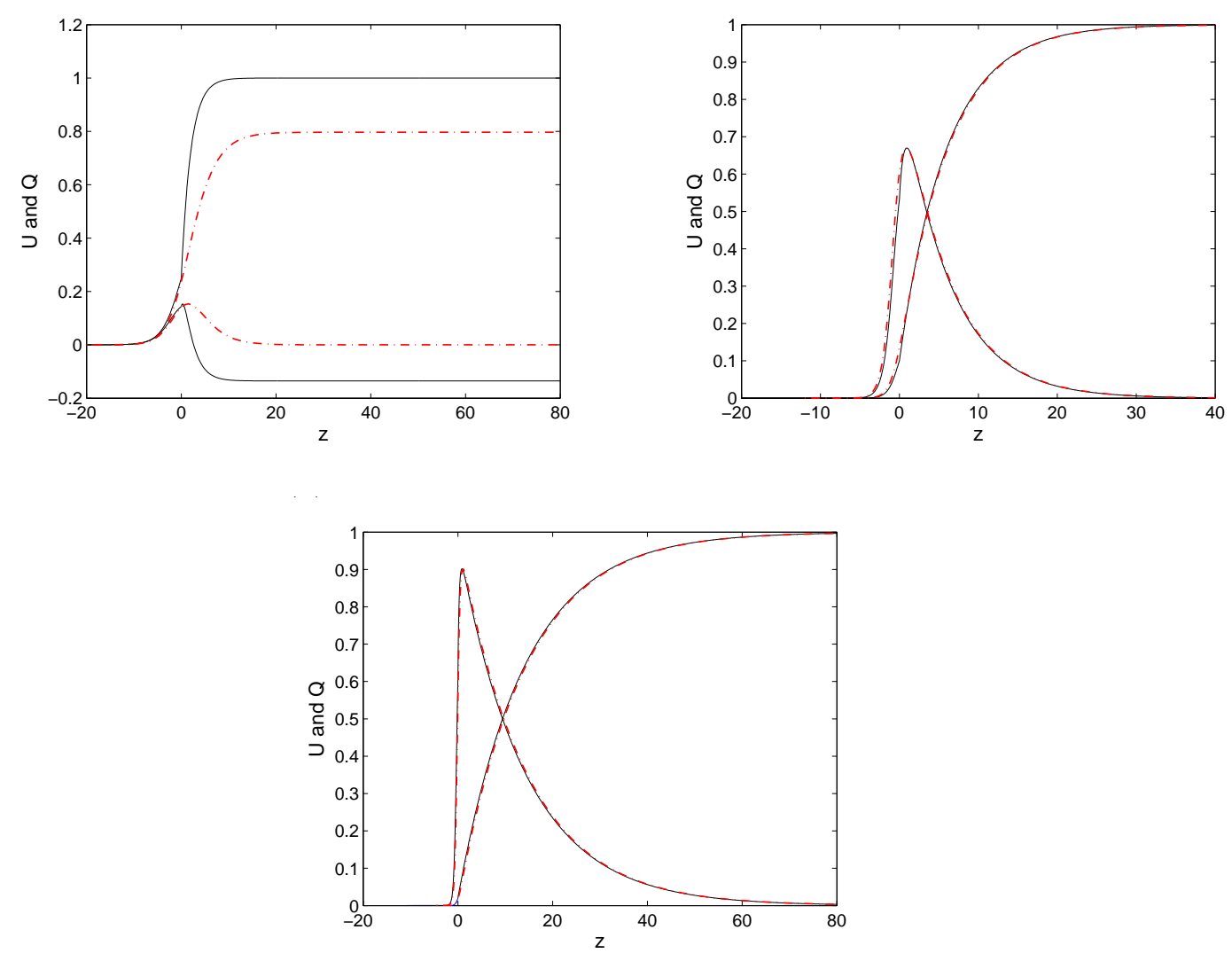

Fig. 17. A comparison between the numerical solution of (40) (black solid line) and the analytical approximation of $Q$ given by (43) and (44) (where $\zeta=\frac{z}{a}$ ) (red dot-dash line). Here $a=2 \sqrt{\alpha-1}$. The approximation is not accurate for small $\alpha$. (a) Here $\alpha=2$. (b) Here $\alpha=10$. (c) Here $\alpha=50$.

\subsection{Approximate Upper Bound for $U$}

The maximum $U$ value has been discussed in Section 5.3 and hence for this case where $C_{2}=-1$ we obtain

$$
U_{\max }=1-\frac{\ln \alpha}{\alpha}-\frac{1}{\alpha},
$$

as an approximation to the original maximum $u$ value.

Figure 18 compares the numerical maximum value of $U$ obtained from the PDE's and the approximation (45) for $1.1 \leq \alpha \leq 10$, which can also be seen in Table A.1 in the Appendix, and for $1.1 \leq \alpha \leq 100$. As can be seen from these figures, this approximation to the maximum value of $u$ is very close to that obtained numerically.

Table A.1 shows that for $\alpha \gtrsim 6$ the approximation equation (34) with $C=1.1$ actually is better for larger $\alpha$ than that of equation (45) where $C=1$. Again 
this shows the small, but non-vanishing, correction due to Region (2).
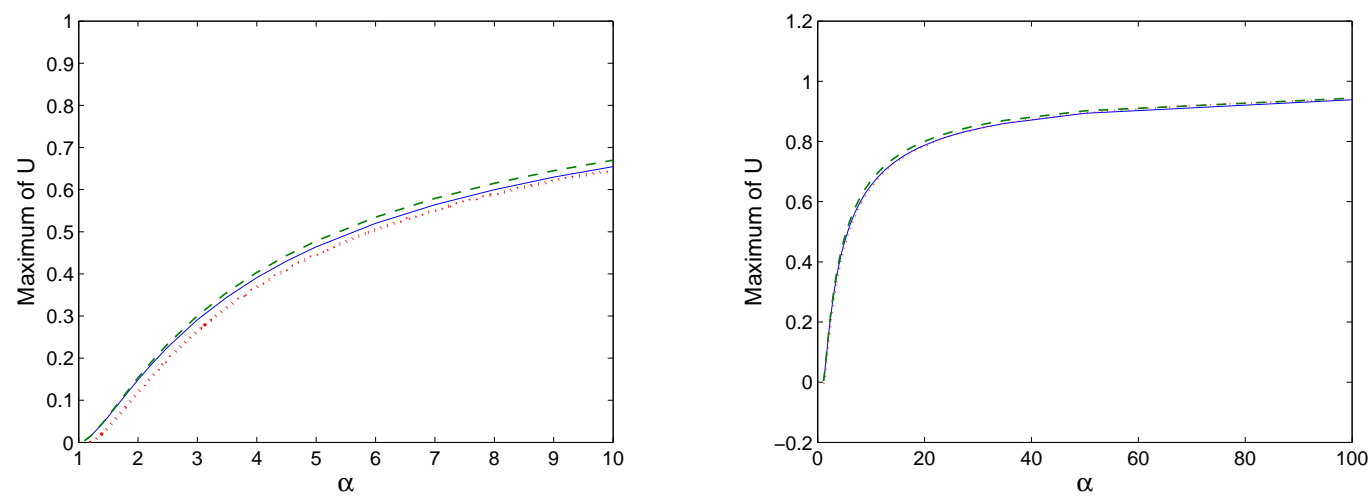

Fig. 18. The blue solid line shows the maximum numerical values of $U$, the green dashed line shows the approximation $U_{\max }=1-\frac{\ln \alpha}{\alpha}-\frac{1}{\alpha}$ and the red dotted line shows the approximation $U_{\max }=1-\frac{1.1 \ln \alpha}{\alpha}-\frac{1}{\alpha}$. It can be seen that the approximation $U_{\max }=1-\frac{\ln \alpha}{\alpha}-\frac{1}{\alpha}$ is better for $\alpha \lesssim 6$ while the other approximation is better for $\alpha \gtrsim 6$. (a) This figure shows the approximation for $1.1 \leq \alpha \leq 10$. (b) This figure shows the approximation for $1.1 \leq \alpha \leq 100$.

\subsection{Maximum Slope of $Q$}

We can find an approximation for the slope of $Q$ with the knowledge that the slope $=\frac{d Q}{d z}=\frac{1}{a} U$. The maximum slope of $Q$ is therefore the maximum of $U$ divided by $a$. Using the approximations (45) and (17), the maximum slope of $Q$ is

$$
Q_{\text {MaxSlope }} \approx \frac{\alpha-\ln \alpha-1}{2 \alpha \sqrt{\alpha-1}} .
$$

A plot of the maximum slope versus $\alpha$ is shown in Figure 19 and this is compared with the numerical maximum slope obtained from the PDE's. It can be seen that the slope increases until $\alpha$ is approximately 5.5 and then decreases. This is in contrast to Murray (2002) who investigated the corresponding slope for the Fisher equation and determined that the wave front becomes less steep as the wave moves faster. In our model this is only true for $\alpha$ greater than approximately $5.5\left(\frac{d Q_{\text {MaxSlope }}}{d \alpha}=5.4861\right)$. The switch in choice of optimal $C$, from $C=1$ to $C=1.1$ occurs at about the same value $\alpha \approx 6$.

\subsection{Approximate Upper Bound for $Q$}

From the approximation (39) we can derive approximate values for $Q(\infty)=N$. As $z \rightarrow \infty$ far behind the wave, $U \rightarrow 0$ and from (39), $N \rightarrow 1-e^{-\alpha N}$. Note that for $\alpha$ very large, $e^{-\alpha N} \approx 0$ and therefore $N \rightarrow 1$. 


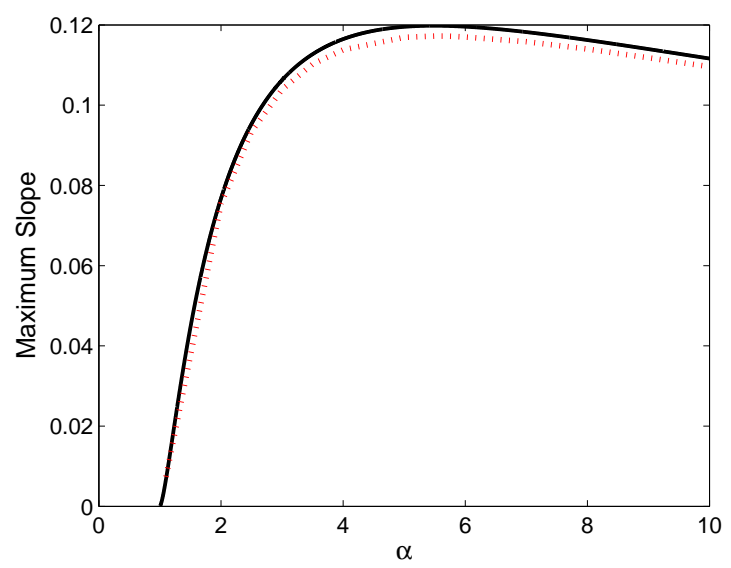

Fig. 19. A graph of the maximum slope of $Q$ versus $\alpha$. The maximum slope determined from the PDE's at $x=-100$ (red dotted line) is compared to the approximate maximum slope $\frac{\alpha-\ln \alpha-1}{2 \alpha \sqrt{\alpha-1}}$ (black solid line). The approximation is reasonably accurate.

Table A.2 in the Appendix shows, for different $\alpha$ values, a comparison between the numerical value of $N$ far behind the wave and the approximations of $N$ obtained from solving $1-N-e^{-\alpha N}=0$. These values are consistent with the constraint $N>1-\frac{1}{\alpha}$. Figure 20 shows these results graphically. It can be seen from the numerical results that $N$ values are above unity for $\alpha$ large.

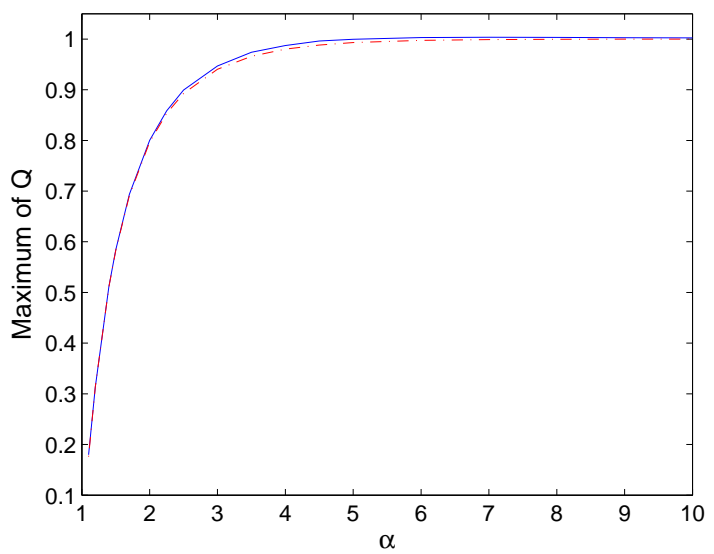

Fig. 20. Blue solid line shows the maximum value of $Q$ obtained from the full numerical solutions of the PDE's and the red dot-dashed line shows the approximation obtained from solving $1-N-e^{-\alpha N}=0$ for $N$ with $1.1 \leq \alpha \leq 10$.

\subsection{Approximation of $Q(\infty)=N$ Using Perturbation Techniques}

We now seek a more accurate approximation for $Q(\infty)=N$. In Section 5 we used the transformation $\zeta=\frac{z}{a}$ on the travelling wave equations (6) and (7) to 
obtain equations (24) and (25). Substituting equation (7) into (6) we obtain

$$
\ddot{Q}(\zeta)=\epsilon \dddot{Q}(\zeta)+\alpha \dot{Q}(\zeta)(1-\dot{Q}(\zeta)-Q(\zeta))-\dot{Q}(\zeta)
$$

where $\epsilon=\frac{1}{a^{2}} \ll 1$. For $\alpha<2, \epsilon>\frac{1}{4}$ which means the approximation is less accurate due to $\epsilon$ being quite large.

Applying perturbation techniques, we substitute

$$
Q=Q_{0}+\epsilon Q_{1}+\epsilon^{2} Q_{2}+O\left(\epsilon^{3}\right)
$$

into equation (46).

At zeroth order we obtain

$$
\ddot{Q}_{0}=\alpha \dot{Q}_{0}\left(1-\dot{Q}_{0}-Q_{0}\right)-\dot{Q}_{0} \text {. }
$$

This is equivalent to solving the system of equations (37) and (38) which results in the first order differential equation $\dot{Q}_{0}=1-Q_{0}-e^{-\alpha Q_{0}}$ which can not be solved analytically for $\zeta$.

At first order we obtain

$$
\ddot{Q_{1}}=\dddot{Q}_{0}+\alpha \dot{Q_{0}}\left(-\dot{Q_{1}}-Q_{1}\right)+\alpha \dot{Q_{1}}\left(1-\dot{Q_{0}}-Q_{0}\right)-\dot{Q_{1}} \text {. }
$$

Since we are interested in the solution as $z \rightarrow \infty$, we allow $\dot{Q}_{1}, \ddot{Q}_{1} \rightarrow 0$. We hence obtain $Q_{1}$ in terms of $Q_{0}$, namely $Q_{1}=\frac{\dddot{Q}_{0}}{\alpha \dot{Q}_{0}}$ as $z \rightarrow \infty$. Using $\dot{Q}_{0}=1-Q_{0}-e^{-\alpha Q_{0}}$ we obtain $\dddot{Q}_{0}=\dot{Q}_{0}\left(1-2 \alpha e^{-\alpha Q_{0}}-\alpha^{2} \dot{Q}_{0} e^{-\alpha Q_{0}}+\alpha^{2} e^{-2 \alpha Q_{0}}\right)$. As $z \rightarrow \infty$ we allow $\dot{Q}_{0} \rightarrow 0$ and hence $e^{-\alpha Q_{0}}=1-Q_{0}$. Therefore, $Q_{1}=\frac{\dddot{Q}_{0}}{\alpha \dot{Q}_{0}}=$ $\frac{1}{\alpha}\left(1-\alpha\left(1-Q_{0}\right)\right)^{2}$ where $Q_{0}$ is the solution to $Q_{0}=1-e^{-\alpha Q_{0}}$.

Recall that $Q=Q_{0}+\epsilon Q_{1}+O\left(\epsilon^{2}\right)$. Replacing $a^{2}=4(\alpha-1)$, we can conclude that

$$
N=Q(\infty) \approx Q_{0}+\frac{\left(1-\alpha\left(1-Q_{0}\right)\right)^{2}}{4(\alpha-1) \alpha},
$$

where $Q_{0}$ is the solution to $Q_{0}=1-e^{-\alpha Q_{0}}$.

The solution to equation (47) for different $\alpha$ values is shown in Table A.2 in the Appendix. It can be seen that the approximation is very accurate for $\alpha$ large but not as accurate for small $\alpha$. The approximation $N=1-e^{-\alpha N}$ is more accurate for $\alpha \lesssim 6$ and then the approximation $N=Q(\infty) \approx Q_{0}+\frac{\left(1-\alpha\left(1-Q_{0}\right)\right)^{2}}{4(\alpha-1) \alpha}$ where $Q_{0}$ is the solution to $Q_{0}=1-e^{-\alpha Q_{0}}$, is more accurate for $\alpha \gtrsim 6$. 


\section{Conclusion}

We have investigated the dynamics of a simplified model of the growth pattern of cells that are sprayed onto a burn to facilitate the healing process. In this model there are two species, one of which migrates into the burn space, proliferates and undergoes a transition to a quiescent cell type that neither migrates nor proliferates. The proliferation of the active cells is modulated by contact inhibition.

The numerical solution of the governing partial differential equations reveals that the invading pulse of active cells and the resulting wave of quiescent cells appears to show a travelling wave structure. The stable invading wave of healing cells travels at the slowest possible speed $a=2 \sqrt{\alpha-1}$ in terms of the parameter $\alpha=k u_{0} / \lambda$. A linear stability analysis about the steady states gives a lower bound on the wave speed in terms of the dimensionless maximum proliferation rate of the active cells, $\alpha$. A further investigation of the travelling wave equations leads to an approximate solution, $\dot{Q}=1-Q-e^{-\alpha Q}$, which in turn leads to estimates of the maximum nondimensional active cell density as well as the final nondimensional cell density within the burn. The maximum active cell density approximation, $U_{\max }=1-\frac{1.1 \ln \alpha}{\alpha}-\frac{1}{\alpha}$, is best for $\alpha \gtrsim 6$ and the approximation, $U_{\max }=1-\frac{\ln \alpha}{\alpha}-\frac{1}{\alpha}$, is best for $1<\alpha \lesssim 6$.

Several approaches are used to obtain improvements to the estimate of the final cell density using perturbation techniques. These are compared with the results obtained from the numerical solution of the original partial differential equations and the approximations are good for large $\alpha$; for $\alpha \gtrsim 6$ we have accuracy to better than the third decimal place using $N=Q(\infty) \approx Q_{0}+$ $\frac{\left(1-\alpha\left(1-Q_{0}\right)\right)^{2}}{4(\alpha-1) \alpha}$ where $Q_{0}$ is the solution to $Q_{0}=1-e^{-\alpha Q_{0}}$. For $\alpha \lesssim 6$ we find $N$ to $1 \%$ accuracy by using $N=1-e^{-\alpha N}$. The dimensional quantities are obtained by multiplying $U_{\max }$ and $N$ by $u_{0}$.

The switch value of approximately $\alpha=6$ arises from the maximum of the graph of the active cell density $U$ divided by the wave speed $a$ (which amounts to the maximum of the quiescent cell density) plotted against $\alpha$, as given in Figure 19.

In this paper we investigated a problem related to the healing of wounds from burns. From our model analysis we derived a set of travelling wave equations which yielded an interesting mathematical analysis. 


\section{Acknowledgements}

The authors thank Dr Graeme Pettet for helpful conversations and Beth Addison-Smith for assistance in computing programming. The results presented here will make up a portion of the doctoral thesis by Paula Voges who has been supported by an Australian Postgraduate Award (APA) and the Tissue Repair and Regeneration Domain in the School of Life Sciences and the Applicable Mathematics and Advanced Computation Program in the School of Mathematical Sciences at Queensland University of Technology. We would like to thank the anonymous referees for their helpful comments that have improved the presentation of this paper.

\section{A Tables}

\section{References}

Barrandon, Y., August 1993. The Epidermal Stem Cell: An Overview. Seminars in Developmental Biology 4 (4), 209-215.

Chalumeau, M., Saulnier, J.-P., Ainaud, P., Lebever, H., Stephanazzi, J., Lecoadou, A., Carsin, H., April 1999. Initial General Management and Surgery of Six Extensively Burned Children Treated With Cultured Epidermal Autografts. Journal of Pediatric Surgery 34 (4), 602-605.

DeLuca, M., Albanese, E., Bondanza, S., Megna, M., Ugozzoli, L., Molina, F., Cancedda, R., Santi, P. L., Bormioli, M., Stella, M., October 1989. Multicentre Experience in the Treatment of Burns with Autologous and Allogenic Cultured Epithelium, Fresh or Preserved in a Frozen State. Burns 15 (5), 303-309.

Fraulin, F. O. G., Bahoric, A., Harrop, A. R., Hiruki, T., Clarke, H. M., 1998. Autotransplantation of Epithelial Cells in the Pig Via an Aerosol Vehicle. The Journal of Burn Care and Rehabilitation 19, 337-345.

Gaffney, E. A., Maini, P. K., Sherratt, J. A., Tuft, S., 1999. The Mathematical Modelling of Cell Kinetics in Corneal Epithelial Wound Healing. Journal of Theoretical Biology 197, 15-40.

Gallico, G. G., O'Connor, N. E., Compton, C. C., Kehinde, O., Green, H., 1984. Permanent Coverage of Large Burn Wounds with Autologous Cultured Human Epithelium. The New England Journal of Medicine 311, 448.

Green, H., November 1991. Cultured Cells for the Treatment of Disease. Scientific American 265 (5), 96-102.

Green, H., Kehinde, O., Thomas, J., November 1979. Growth of Cultured Human Epidermal Cells into Multiple Epithelia Suitable for Grafting. Proceedings of the National Academy of Sciences of the United States of America 76 (11), 5665-5668. 


\begin{tabular}{|c|c|c|c|c|}
\hline$\alpha$ & PDE Numerics & Approx. $C=1.1$ & Approx. $C=1$ & TWE's \\
\hline 1.1 & 0.0042 & -0.0044 & 0.00426 & $0.0042(N=0.17616)$ \\
2 & 0.1489 & 0.1188 & 0.1534 & $0.1487(N=0.80024)$ \\
3 & 0.2911 & 0.2638 & 0.3005 & $0.291(N=0.947)$ \\
4 & 0.3911 & 0.3688 & 0.4034 & $0.391(N=0.9874)$ \\
5 & 0.4643 & 0.4459 & 0.4781 & $0.4645(N=0.9998)$ \\
6 & 0.5201 & 0.5048 & 0.5347 & $0.5209(N=1.00335)$ \\
7 & 0.5641 & 0.5514 & 0.5792 & $0.564(N=1.00395)$ \\
8 & 0.5999 & 0.5891 & 0.6151 & $0.6003(N=1.00366)$ \\
9 & 0.6296 & 0.6203 & 0.6448 & $0.6312(N=1.00315)$ \\
10 & 0.6547 & 0.6467 & 0.6697 & $0.6551(N=1.00265)$ \\
20 & 0.7874 & 0.7852 & 0.8002 & $0.7873(N=1.00066)$ \\
35 & 0.8598 & 0.8597 & 0.8698 & $0.8601(N=1.00021)$ \\
50 & 0.8935 & 0.8939 & 0.9018 & $(N=1.00010)$ \\
100 & 0.9385 & 0.9393 & 0.9439 & $(N=1.000025)$ \\
\hline
\end{tabular}

Table A.1

The maximum of $u$ and $U$ from (a) examining the numerical solutions of equations (4) and (5), (b) the approximated equation (39) where the maximum is at $U_{\max }=$ $1-\frac{\ln \alpha}{\alpha}-\frac{1}{\alpha}$, (c) the approximation $U_{\max }=1-\frac{1.1 \ln \alpha}{\alpha}-\frac{1}{\alpha}$, (d) the numerical solutions to the travelling wave equations. Note that for $1<\alpha \lesssim 6$ the $C=1$ approximation is accurate to about 0.01 , while for $6 \lesssim \alpha \leq 100$ we note that the $C=1.1$ approximation is accurate to about 0.01 or better. The value of $N$ used when solving the travelling wave equations is given to 5 decimal places, however, for $\alpha \geq 10$, we required $N$ to have over ten decimal place accuracy to shoot successfully. Note that for $\alpha \geq 50, N$ requires an accuracy of at least 50 decimal places, and we have not found the maximum $U$ for the travelling waves.

Harris, P. A., Leigh, I. M., Navsaria, H. A., 1998. Pre-Confluent Keratinocyte Grafting: The Future for Cultured Skin Replacements? Burns 24, 591-593. Maini, P. K., McElwain, D. L. S., Leavesley, D. I., 2004. Travelling Waves in a Wound Healing Assay. Applied Mathematics Letters 17 (5), 575-580.

Murray, J. D., 2002. Mathematical Biology I: An Introduction, 3rd Edition. Vol. 1. Springer-Verlag.

O'Connor, N. E., Mulliken, J. B., Banks-Schlegel, S., Kehinde, O., Green, H., 1981. Grafting of Burns with Cultured Epithelium Prepared from Autologous Epidermal Cells. The Lancet, 1-75.

Pettet, G. J., Byrne, H. M., McElwain, D. L. S., Norbury, J., 1996a. A Model of Wound Healing Angiogenesis in Soft-Tissue. Mathematical Biosciences 136, 35-64.

Pettet, G. J., Chaplain, M. A., McElwain, D. L. S., Byrne, H. M., 1996b. 


\begin{tabular}{|c|c|c|c|}
\hline$\alpha$ & Numerical $N$ & Approximated $N$ & $N$ from Perturbations \\
\hline 1.1 & 0.1761 & 0.1761 & 0.1961 \\
2 & 0.8003 & 0.7968 & 0.8409 \\
3 & 0.9471 & 0.9405 & 0.9686 \\
4 & 0.9875 & 0.9802 & 0.9978 \\
5 & 0.9999 & 0.993 & 1.0047 \\
6 & 1.0034 & 0.9975 & 1.0056 \\
7 & 1.004 & 0.9991 & 1.0050 \\
8 & 1.0037 & 0.9997 & 1.0041 \\
9 & 1.0032 & 0.9999 & 1.0033 \\
10 & 1.0027 & 1 & 1.0027 \\
20 & 1.0006 & 1 & 1.0007 \\
50 & 1.0001 & 1 & 1.0001 \\
\hline
\end{tabular}

Table A.2

A comparison of the numerical value of $N$ behind the wave, the approximated values of $N$ behind the wave, $N=1-e^{-\alpha N}$, and the approximation of $N$ using perturbation techniques, $N=Q(\infty) \approx Q_{0}+\frac{\left(1-\alpha\left(1-Q_{0}\right)\right)^{2}}{4(\alpha-1) \alpha}$ where $Q_{0}$ is the solution to $Q_{0}=1-e^{-\alpha Q_{0}}$, for different $\alpha$. Note that the approximation $N=1-e^{-\alpha N}$ is most accurate for $\alpha \lesssim 6$ while the approximation for $N$ using the perturbation technique is most accurate for $\alpha \gtrsim 6$.

On the Role of Angiogenesis in Wound Healing. Proceedings of the Royal Society of London B 263, 1487-1493.

Sherratt, J. A., 2000. Wavefront Propagation in a Competition Equation with a New Motility Term Modelling Contact Inhibition Between Cell Populations. Proceedings of the Royal Society of London A 456, 2365-2386.

Voges, P. K., McElwain, D. L. S., Harkin, D. G., Upton, Z., 2006. Mathematical Modelling of Aerosolised Skin Grafts Incorporating Keratinocyte Clonal Subtypes. Submitted.

Williamson, J. S., Snelling, C. F. T., Clugston, P., Macdonald, I. B., Germann, E., 1995. Cultured Epithelial Autograft: Five Years of Clinical Experience with Twenty-Eight Patients. The Journal of Trauma: Injury, Infection, and Critical Care 39 (2), 309-319.

Wood, F., 2003. Clinical Potential of Autologous Epithelial Suspension. Wounds 15 (1), 16-22. 